\title{
Early error detection predicted by reduced pre-response control process: an ERP topographic mapping study
}

\author{
Gilles Pourtois \\ Department of Experimental Clinical and Health Psychology, Ghent University, Belgium
}

Running head: Error detection predicted by reduced control process

Correspondence :

Gilles Pourtois

Department of Experimental Clinical and Health Psychology

Ghent University

Henri Dunantlaan 2

9000 Gent

Belgium

Phone: +3292649144

Email: gilles.pourtois@ugent.be 


\begin{abstract}
Advanced ERP topographic mapping techniques were used to study error monitoring functions in human adult participants, and test whether proactive attentional effects during the pre-response time period could later influence early error detection mechanisms (as measured by the ERN component) or not. Participants performed a speeded go/nogo task, and made a substantial number of false alarms that did not differ from correct hits as a function of behavioral speed or actual motor response. While errors clearly elicited an ERN component generated within the dACC following the onset of these incorrect responses, I also found that correct hits were associated with a different sequence of topographic events during the pre-response baseline time-period, relative to errors. A main topographic transition from occipital to posterior parietal regions (including primarily the precuneus) was evidenced for correct hits $\sim 170-150 \mathrm{~ms}$ before the response, whereas this topographic change was markedly reduced for errors. The same topographic transition was found for correct hits that were eventually performed slower than either errors or fast (correct) hits, confirming the involvement of this distinctive posterior parietal activity in top-down attentional control rather than motor preparation. Control analyses further ensured that this pre-response topographic effect was not related to differences in stimulus processing. Furthermore, I found a reliable association between the magnitude of the ERN following errors and the duration of this differential precuneus activity during the pre-response baseline, suggesting a functional link between an anticipatory attentional control component subserved by the precuneus and early error detection mechanisms within the dACC. These results suggest reciprocal links between proactive attention control and decision making processes during error monitoring.
\end{abstract}

Keywords: error detection; ERN; dACC; proactive attentional control; topographic ERP mapping; precuneus 


\section{Introduction}

Error detection plays a critical role in action regulation and cognitive control (Carter, Botvinick, \& Cohen, 1999). The commission of (unwanted) errors has been repeatedly associated with specific ERP components following motor response, including the ErrorRelated Negativity (ERN) and the Positivity error (Pe, see Falkenstein, Hoormann, Christ, \& Hohnsbein, 2000; Holroyd \& Coles, 2002; Ullsperger \& von Cramon, 2001 for overviews). The ERN component is a negative brain potential with a fronto-central scalp distribution (maximum amplitude at FCZ electrode position), peaking early following the onset of erroneous motor responses (typically in a window spanning from 0 until $100 \mathrm{~ms}$ after incorrect key presses). The neural generators of the ERN have been consistently localized in the dorsal Anterior Cingulate Cortex across several studies (dACC, see Dehaene, Posner, \& Tucker, 1994; Bush, Luu, \& Posner, 2000; Debener et al., 2005; van Veen \& Carter, 2006; Pizzagalli, Peccoralo, Davidson, \& Cohen, 2006; Ridderinkhof, Nieuwenhuis, \& Braver, 2007; Vocat, Pourtois, \& Vuilleumier, 2008; Pourtois et al., 2010). Time-frequency analyses showed that the ERN was primarily driven by transiently phase-locked theta-band power increase to the subject's motor response (Luu, Tucker, \& Makeig, 2004).

Based on these robust electrophysiological properties, it was initially proposed that the ERN primarily indexes an early (cognitive) mismatch process between the intended or desired and actual response (Falkenstein, Hohnsbein, Hoormann, \& Blanke, 1991; Coles, Scheffers, \& Holroyd, 2001; Nieuwenhuis, Ridderinkhof, Blom, Band, \& Kok, 2001). Other alternative theoretical accounts suggested that the ERN reflects either mechanisms of reinforcement learning implicating dopaminergic midbrain structures (Holroyd \& Coles, 2002; Nieuwenhuis, Holroyd, Mol, \& Coles, 2004) or conflict monitoring processes (Carter et al., 1998; van Veen, Cohen, Botvinick, Stenger, \& Carter, 2001; Yeung, Cohen, \& Botvinick, 2004). Therefore, the ERN component is assumed to be a reliable ERP marker of an early (perhaps automatic in the sense of unconscious, see Nieuwenhuis et al., 2001) and online detection of errors (recruiting primarily the cingulate motor area, see Ullsperger \& von Cramon, 2001), based either on a rapid matching process (Scheffers, Coles, Bernstein, Gehring, \& Donchin, 1996), on a reinforcement learning mechanism (Holroyd \& Coles, 2002), or on conflict monitoring (Botvinick, Braver, Barch, Carter, \& Cohen, 2001; Yeung et al., 2004).

Whereas the ERN is defined as an ERP component being primarily time-locked and, to a lesser degree, phased-locked to the subject's motor response ("response-triggered component", see Coles et al., 2001; Luu et al., 2004), an intriguing possibility is that the commission of errors may also depend, to some extent, on other higher-level (endogenous) attentional factors, some of which actually take place before the subject overtly committed an error (i.e., during the pre-response time period, which foregoes the motor preparation and execution stages, sharing similarities with an anticipatory or proactive attentional component, see Weissman, Roberts, Visscher, \& Woldorff, 2006; Orr \& Weissman, 2009). Under increased attentional demands, there is a need for strong top-down biasing attentional resources (Desimone \& Duncan, 1995) and it seems therefore plausible to surmise that errors, under some circumstances (e.g., when the task demands require a high level of attentional 
control on a trial by trial basis, as in the present case, see Vocat et al., 2008), may also be partly explained by a deficiency in higher-order attentional control mechanisms, which actually take place sometime before these incorrect motor responses occur, consistent with the notion of proactive attentional control processes (see Braver et al., 2007; Braver et al., 2009). In agreement with this view, several reports have already revealed distinct attentional "preparatory sets" that preceded, and sometimes even predicted, the accuracy (and speed) of motor behavior during simple visuo-motor tasks (Gevins et al., 1987; Gratton, Coles, Sirevaag, Eriksen, \& Donchin, 1988; Coles, Gratton, \& Donchin, 1988). Likewise, more recent EEG studies have also shown a positive modulation of the response-locked ERP ("error preceding positivity") on trials preceding errors (Ridderinkhof, Nieuwenhuis, \& Bashore, 2003; Allain, Carbonnell, Falkenstein, Burle, \& Vidal, 2004; Hajcak, Nieuwenhuis, Ridderinkhof, \& Simons, 2005). This latter ERP activity was thought to reflect a transient deficiency in the action monitoring system. Moreover, in another ERP study (Padilla, Wood, Hale, \& Knight, 2006), error trials during a letter discrimination task were mainly characterized by a decreased CNV prior to stimulus presentation, compatible with transient lapses in a preparatory attention network that foreshadow response errors (see also Mazaheri, Nieuwenhuis, van Dijk, \& Jensen, 2009 for MEG evidence). Finally, several fMRI studies have also shown that changes in activity in default mode regions of the brain preceded and even predicted performance errors (Li, Yan, Bergquist, \& Sinha, 2007; Eichele et al., 2008). Altogether, these studies confirm that pre-response attentional fluctuations may foreshadow response errors. Moreover, recent findings suggest that the posterior parietal cortex (e.g. the precuneus) may play an important role in these pre-response attentional fluctuations, as reviewed in the next section.

Electrophysiological studies have demonstrated that the DorsoLateral Prefrontal Cortex (DLPFC) fires when relevant information must be maintained across a delay, during the preparatory period of the task (Cohen et al., 1997; Levy \& Goldman-Rakic, 1999). It was proposed that the dACC directly interacts with the DLPFC when a response conflict (or error) occurs (Botvinick et al., 2001). Within this influential model, the dACC signals the DLPFC of an ongoing response conflict, which in turn increases attentional resources (Ridderinkhof, Ullsperger, Crone, \& Nieuwenhuis, 2004; Orr \& Weissman, 2009). This dACC-DLPFC system has been implicated in tasks that require overcoming a prepotent response tendency (MacDonald, Cohen, Stenger, \& Carter, 2000; Barber \& Carter, 2005). Interestingly, Bunge et al. (2002) suggested that the DLPFC activation may not reflect working-memory load per se (Cohen et al., 1997; Levy \& Goldman-Rakic, 1999), but rather, a selection process between competing responses (see also Rowe, Toni, Josephs, Frackowiak, \& Passingham, 2000); or alternatively an attempt to overcome residual inhibition (see Dreher \& Berman, 2002), while a repertoire of potential S-R associations would be "pre-activated" within posterior parietal cortex presumably at an earlier stage of processing, including regions of the precuneus (Barber \& Carter, 2005). Within this model, the posterior parietal cortex would be activated during the anticipatory period of the task to increase (or maybe to switch) attentional resources towards the relevant stimulus features necessary for upholding S-R associations (Rushworth, Paus, \& Sipila, 2001; Bunge, Hazeltine, Scanlon, Rosen, \& Gabrieli, 2002; Astafiev et al., 2003; Barber \& Carter, 2005; Rushworth \& Taylor, 2006). Consistent with this 
view, Barber and Carter (2005) used fMRI and elegantly demonstrated that the precuneus showed a sustained activation during the anticipatory period of the task, when participants were instructed to overcome a prepotent response tendency (i.e., to use a less frequent and reversed S-R mapping compared to a more intuitive and standard S-R mapping), confirming that posterior parietal regions played a general role in top-down biasing processing resources under increased attentional demands (see also Desimone \& Duncan, 1995; Kastner \& Ungerleider, 2000; Corbetta \& Shulman, 2002; Lavie, 2005; Li et al., 2007).

The goal of this ERP study was to gain further insight into error monitoring functions in human adult participants, and more precisely to address the question whether a differential proactive attentional control effect (see also Braver, Gray, \& Burgess, 2007; Braver, Paxton, Locke, \& Barch, 2009) could be detected between errors (false alarms) vs. correct hits during the pre-response time period (baseline) or not. Importantly, I aimed to compare errors to correct hits, when obvious lapses of vigilance did not account for the commission of these errors (see Vocat et al., 2008; Pourtois et al., 2010). More precisely, I predicted that errors would be associated with the marked attenuation of a distinctive anticipatory component, presumably reflecting top-down or proactive attentional control (therefore recruiting regions of the posterior parietal cortex, such as the precuneus; see Li et al., 2007) and hence indirectly contributing to mechanisms of action monitoring (e.g., involved in readying the cognitive system for task performance under high attentional demands, see Barber \& Carter, 2005). For this purpose, I performed advanced topographic mapping analyses of previously published ERP data (see Vocat et al., 2008). In this earlier ERP study, Vocat et al. (2008) designed a new speeded go/nogo task enabling a direct comparison of two opposite accuracy conditions (correct hits vs. errors), in the absence of significant speed (RT) differences, and when errors were relatively frequent events (as opposed to deviant), compared to correct hits. These two conditions were important pre-requisites to provide a balanced comparison between hits and errors in terms of overall attentional demands, ruling out the possibility that errors would mainly correspond to (deviant) lapses of attention or vigilance in this task. In the study of Vocat et al. (2008), this was mainly achieved by imposing strong time pressure to participants (calibrated and adjusted online separately for each participant throughout the whole experimental session), eventually leading to fast perceptual decisions in all participants, including a high proportion of hits (either fast or slow) and false alarms (always performed as fast as the fastest correct hits, see Vocat et al., 2008). In other words, errors, which were somewhat unavoidable, did not lead to either faster or slower motor responses (RTs) compared to correct (fast) hits in this speeded go/nogo task (Vocat et al., 2008). Hence, the go/nogo task used in this study primarily required the inhibition of a pre-potent response tendency, where proactive attentional mechanisms (operating between stimulus processing and response execution) were presumably involved (Dempster \& Corkill, 1999; Friedman \& Miyake, 2004). In this study, Vocat et al. (2008) could therefore separate ERP components for correct vs. incorrect simple key presses, while attentional demands between these two conditions were nearly equated. Vocat et al. (2008) primarily studied response-related ERP components and reported that errors in this task generated conspicuous ERN and Pe components, relative to correct responses (hits), confirming that the detection of error was 
associated with well-established error-related ERP components in this new speeded go/nogo task (see Falkenstein et al., 2000).

In the present study, I performed new topographic mapping analyses of these ERP data (Vocat et al., 2008), and focused on the $500 \mathrm{~ms}$ time period preceding the registering of correct (hits) vs. incorrect (false alarms) motor responses (RTs). To identify reliable topographic differences between conditions during this pre-response time-period, I used the same procedure for data analysis as already described in previous ERP topographic mapping studies (see Pourtois, Thut, Grave de Peralta, Michel, \& Vuilleumier, 2005; Pourtois, Dan, Grandjean, Sander, \& Vuilleumier, 2005; Pourtois, De Pretto, Hauert, \& Vuilleumier, 2006; see Murray, Brunet, \& Michel, 2008; Pourtois, Delplanque, Michel, \& Vuilleumier, 2008 for a detailed presentation of the basic principles of this method). Notably, this topographic mapping method was already used to reveal substantial ERP topographic changes across experimental conditions occurring during the pre-stimulus (baseline) time period (see Kondakor, Pascual-Marqui, Michel, \& Lehmann, 1995; Pourtois et al., 2006), when the amplitude (strength) of the ERP signal is usually low (close to zero baseline) and therefore where conventional ERP techniques (peak analyses, see Picton et al., 2000) usually fail to disclose reliable differences between experimental conditions (see Pourtois et al., 2008 for a thorough discussion). In this study, I first identified global ERP differences between conditions and distinguished between global differences due to (1) variations in field strength and (2) topography based on the reference-free global field power and the global spatial dissimilarity indices, respectively (Lehmann \& Skrandies, 1980). I then performed (3) a detailed temporal segmentation analysis for each of the experimental conditions to characterize the precise spatio-temporal sequence of electric field configurations from -500 ms until response onset (R.D. Pascual-Marqui, Michel, \& Lehmann, 1995; C.M. Michel, Seeck, \& Landis, 1999; C. M. Michel et al., 2001). Finally, I applied (4) a linear distributed source localization technique (i.e., Standardized low-resolution brain electromagnetic tomography, sLORETA, R. D. Pascual-Marqui, 2002) to determine brain regions that might generate the topographic patterns observed in each experimental condition.

\section{Methods}

\section{Participants}

Sixteen healthy participants (9 women) with a mean age of 27 years (S.D. $=2)$ took part in the present study. They reported no history of neurological or psychiatric disease and normal or corrected-to-normal vision. The study was approved by the local university ethical committee.

\section{Stimuli and task}

Extensive details regarding the stimuli, task parameters and RT calibration procedure used in this experiment can be found in Vocat et al. (2008) and Pourtois et al. (2010). 
Visual stimuli consisting of simple arrow symbols were presented centrally, and were oriented either upward or downward. Each trial started with a black arrow (upright or inverted), presented centrally for a variable duration of 1000-2000 ms. The black arrow was then immediately replaced by a colored arrow (green or turquoise) at the same central location, but with either the same or the opposite orientation. These different combinations of color and orientation were used as imperative cues for the Go/noGo response. Notably, the task was initially designed in such a way to minimize low-level differences between go and nogo trials (see Vocat et al., 2008). For each and every trial, a changing arrow was always shown (after an initial black arrow), whose color and orientation features precisely indicated the response to be made (a fine-grained color + orientation discrimination was thus required). Hence, the amount of perceptual change (at the level of the changing arrow) was actually balanced between go and nogo trials. The colored arrow remained on the screen until the subject's response (on Go trials) or for a maximum of $1500 \mathrm{~ms}$ (on noGo trials). The intertrial intervals (ITI) included a blank screen of $500 \mathrm{~ms}$, followed by a central fixation cross presented for another $500 \mathrm{~ms}$.

Participants were instructed to perform a speeded color plus orientation discrimination task. They had to press the response key as fast as possible if the black arrow turned green and kept the same orientation (Go trials). By contrast, they were asked not to respond if the black arrow turned green but changed orientation, or if it turned turquoise irrespective of orientation (noGo trials). In addition, they were asked to verbally report their errors, if they felt they had committed a response error.

The experiment was divided into three sessions, each starting with a calibration block (containing 14 trials: 10 Go and 4 noGo), immediately followed by two consecutive test blocks (containing 60 trials each: 40 Go and 20 noGo). Only ERPs recorded during test blocks $(n=6)$ were used for subsequent data analyses. Trial presentation was randomized within blocks. During each calibration block, the mean RT for Go trials was calculated online and used to define an upper limit for correct Go trials in the subsequent test blocks (see Vocat et al., 2008 for additional details). Participants received feedback about their speed of decision during the test blocks (on Go trials). When a correct response to a Go trial was made with RT above the upper limit, a feedback screen was displayed (with the words "Too late" in a red frame, for $500 \mathrm{~ms}$ ), immediately following the response (these correct trials were subsequently classified as "Slow Hits"). "Fast Hits" corresponded to correct responses to Go trials made below the upper limit. The speed pressure imposed by this procedure promoted the occurrence of many errors, consisting of false alarms on the noGo trials (subsequently classified as "Errors"). The whole experiment lasted on average 20 minutes.

\section{EEG recording}

Continuous scalp EEG was acquired at $2048 \mathrm{~Hz}$ (0-417 Hz band-pass) using a 64-channel (pin-type) Biosemi ActiveTwo system (http://www.biosemi.com) referenced to the CMSDRL ground (driving the average potential across the montage as close as possible to the amplifier zero). Details of this circuitry can be found on the Biosemi website 
(http://www.biosemi.com/faq/cms and http://drl.htm). Electrodes were evenly distributed over the scalp according to the extended international 10-20 EEG system. Three electrodes (P9, Iz and P10) had more eccentric lower positions relative to the 61 other electrodes forming a uniform spherical head model, and these three electrodes were therefore not included in the subsequent ERP data analyses. EEG data were first downsampled to $512 \mathrm{~Hz}$. ERPs of interest were computed offline following a standard sequence of data transformation (Picton et al., 2000): (1) common average reference, (2) ocular correction for blinks (Gratton, Coles, \& Donchin, 1983) using the electrode FP1, (3) -500/+500 ms epoching around either the stimulus or the motor response onset time, (4) pre-response (or pre-stimulus) interval baseline correction (from $-500 \mathrm{~ms}$ to either motor response or stimulus onset), (5) artifact rejection (mean of $-52.5 /+52.5 \mathrm{mV}$ amplitude scale across participants), (6) averaging for each of the 3 critical experimental conditions (Fast Hits, Slow Hits, and Errors), and (7) $30 \mathrm{~Hz}$ low-pass digital filtering of the individual average data. Several auxiliary analyses confirmed that the use of a $100 \mathrm{~ms}$, instead of a $500 \mathrm{~ms}$, pre-response baseline correction did not substantially alter the pre-response topographic shift observed for hits, which was strongly reduced for errors (see results here below).

\section{ERP data analyses}

A detailed presentation of ERP components following the onset of the response (including the ERN and Pe components generated in response to errors, and their respective topographic properties) can be found in Vocat et al. (2008).

In this study, I focused on ERP effects occurring during the $500 \mathrm{~ms}$ pre-response time period. Because I primarily focus on pre-response attentional changes likely occurring before the onset of the response, I selected on purpose this broad temporal interval, spanning from a 500 to $0 \mathrm{~ms}$ relative to the onset of the response and encompassing most of the stimuluslocked effects (see results section). Moreover, a similar pre-response interval was used in previous ERP studies focused on early error-detection brain mechanisms (Vocat et al., 2008; Pourtois et al., 2010). Importantly, I also performed additional analyses using the stimulus (i.e. the changing/color arrow, see stimuli and task) as the reference point to establish whether the attentional effects found during the pre-response baseline (see results section) were actually related to systematic differences during early stimulus processing across conditions or not.

In order to capture potential differences between errors and correct hits (with a focus on fast hits for which the RT speed was comparable to errors, see behavioral results here below) during the $500 \mathrm{~ms}$ time period before motor response (RT) where the amplitude of the ERP signal was by definition low (and thus where systematic ERP components could be hardly detected with confidence, see Picton et al., 2000), I performed a detailed topographic mapping analysis of the pre-response ERP data, following a conventional four-step procedure (see C.M. Michel et al., 1999; C. M. Michel et al., 2001; Pourtois, Thut et al., 2005; Murray et al., 2008; Pourtois et al., 2008). Noteworthy, it was also important to show that the ERP effects found for fast hits (and reduced for errors) were truly related to "accuracy". I have 
therefore used slow hits as an additional control condition (and hence also directly compared slow hits to errors), although slow hits differed from errors with respect to speed (see behavioral results here below). Two simple contrasts (and a Bonferroni correction) were therefore mainly used in the analyses reported in this study (global field power vs. global dissimilarity), i.e. a main one: Fast hits vs. Errors; an auxiliary one: Slow hits vs. Errors.

(1) Changes in electric field strength were first determined by calculating the global field power (GFP, see Lehmann \& Skrandies, 1980) for each subject and each condition. GFP is equivalent to the spatial standard deviation of the scalp electric field, with larger values for stronger electric fields, and is calculated as the square root of the mean of the squared value recorded at each electrode (vs. the average reference). Reliable changes in the strength of the ERP signal were verified by performing a series of paired non-parametric statistical analyses based on stringent randomization tests (Manly, 1991; see also Pourtois, Peelen, Spinelli, Seeck, \& Vuilleumier, 2007; Pourtois et al., for recent applications to EEG data). The statistical approach used in this study is actually standard, and borrowed from previous work and guidelines for ERP topographic analyses (see Murray et al., 2008). Because there are some uncertainties regarding the exact (statistical) distribution of continuous GFP (and dissimilarity values, see here below), non-parametric statistical analyses were used to test for differences in GFP (or dissimilarity). Randomization provides a robust non-parametric method to test for differences in any variable (here amplitude at each time-point) without any assumptions regarding data distribution, by comparing the observed dataset with random shuffling of the same values over many iterations. The method runs by repeating the shuffling many times (minimum of 5000 with the randomization tests used here) so as to be able to estimate the probability (here $\mathrm{p}<0.01$; with an additional criterion of temporal stability for 5 consecutive time-points, corresponding to $>10 \mathrm{~ms}$ at $512 \mathrm{~Hz}$ sampling rate) that the data might be observed by chance. The selection of a temporal stability of $10 \mathrm{~ms}$ was based on previous EEG studies (Murray et al., 2008; Pourtois et al., 2008). A significant GFP modulation does not exclude the possibility of concurrent changes in field topography, but the observation of a GFP modulation in the absence of a topographic modulation is indicative of amplitude modulation within indistinguishable generators (Lehmann, 1987).

(2) Significant periods of topographic modulation were next determined by calculating the global dissimilarity (Lehmann \& Skrandies, 1980). Global dissimilarity is an index of configuration differences between two electric fields, independent of their strength. Global dissimilarity has been shown to be a reliable measure to identify transitions between dominant topographies (Murray et al., 2008. This parameter equals the square root of the mean of the squared differences between the potentials measured at each electrode (vs. the average reference), each of which is first scaled to unitary strength divided by the instantaneous GFP. Dissimilarity can range from 0 to 2 , where 0 indicates topographic homogeneity and 2 indicates topographic inversion. Two complementary analyses were used with the dissimilarity index. First, for each condition separately (errors, fast and slow hits), increases of dissimilarity were assessed. The onsets of topographic changes were detected by comparing dissimilarity values at a given time point with the value calculated at the preceding time point. Although this statistical approach is valid to identify transient periods of 
topographic changes, other methods have been developed recently to detect the presence of "components" (Koenig \& Melie-Garcia, 2010). Second, changes of dissimilarity between conditions (fast hits vs. errors or slow hits vs. errors) were tested. Unlike changes in GFP, electric field changes may be indicative of changes in the underlying generator configuration (Lehmann, 1987). Consistent changes in the electric field configuration were verified by performing a series of paired nonparametric statistical analyses, based on similar randomization tests as used for the GFP (see point 1 here above).

(3) Topographic analyses based on the global dissimilarity measure (see point 2) are particularly useful to identify significant periods of topographic modulation (Lehmann, 1987). However, the global dissimilarity measure alone is not sufficient to determine whether topographic differences are explained by a single or multiple configuration change, or by a latency shift in a given topography across conditions. To better characterize topographic modulations over time and conditions, I thus applied a pattern or spatial cluster analysis procedure. The pattern analysis efficiently summarizes ERP data by a limited number of field configurations, previously referred to as functional microstates (Lehmann, 1987; C.M. Michel et al., 1999). Here, I performed a topographic pattern analysis on group-averaged data from $500 \mathrm{~ms}$ until response onset (256 time frames at $512 \mathrm{~Hz}$ sampling rate) using a standard cluster (or spatio-temporal segmentation) method (K-means, see R.D. Pascual-Marqui et al., 1995) and then fitted the segmentation results back to individual data for subsequent statistical testing. The rationale and basic principles of this temporal segmentation method have been extensively described elsewhere (see C.M. Michel et al., 1999; Murray et al., 2008). The spatio-temporal segmentation algorithm is derived from spatial cluster analysis (R.D. PascualMarqui et al., 1995) and allows the identification of the most dominant scalp topographies appearing in the group-averaged ERPs of each condition and over time, while minimizing the biases for the selection of time-frames or electrodes of interest. The optimal number of topographic maps explaining the whole data set is determined objectively using both cross validation (R.D. Pascual-Marqui et al., 1995) and Krzanowski-Lai (Tibshirani, Walther, \& Hastie, 2001) criteria. The dominant scalp topographies (identified in the group-averaged data) are then fitted to the ERPs of each individual subject using spatial fitting procedures to quantitatively determine their representation across subjects and conditions. This procedure thus provides fine-grained quantitative values, such as the duration of a specific topographic map or its global explained variance (GEV, or goodness of fit), which are critical indices of the significance of a given topography, not available otherwise in a classical component analysis (Picton et al., 2000). GEV represents the sum of the explained variance weighted by the GFP at each moment in time. Goodness of fit and map duration were entered in repeatedmeasure analyses of variance (ANOVAs) with two within-subject factors: condition (Fast hits, Slow hits or Errors) and map configuration (i.e., the two electric field distributions previously identified by the spatial cluster analysis). Data obtained after the fitting procedure (GEV values) were analyzed using conventional parametric tests (t-tests and ANOVAs) because these data fulfilled the requirements of normality. These analyses were carried out using CARTOOL software (Version 3.34; developed by D. Brunet, Functional Brain Mapping Laboratory, Geneva, Switzerland). Finally, correlation analyses (Pearson correlation coefficient) were also performed on the map duration, enabling to estimate the degree to 
which the length of a given early topography during the pre-response time interval was associated with the magnitude of post-response error-related ERP components, such as the ERN or Pe.

(4) Finally, to estimate the likely neural sources underlying the electrical field configurations identified by the previous analyses, I used a specific distributed linear inverse solution, namely standardized low-resolution brain electromagnetic tomography (sLORETA, R. D. Pascual-Marqui, 2002). sLORETA is based on the neurophysiological assumption of coherent coactivation of neighboring cortical areas (known to have highly synchronized activity, see Silva, Amitai, \& Connors, 1991) and, accordingly, it computes the "smoothest" of all possible activity distributions (i.e. no a priori assumption is made on the number and locations of the sources). Mathematical validation of this distributed source localization technique has been recently demonstrated (Sekihara, Sahani, \& Nagarajan, 2005). sLORETA solutions are computed within a three-shell spherical head model co-registered to the MNI152 template (Mazziotta et al., 2001). The source locations were therefore given as (x,y,z) coordinates ( $\mathrm{x}$ from left to right; $\mathrm{y}$ from posterior to anterior; $\mathrm{z}$ from inferior to superior). SLORETA estimates the 3-dimensional intracerebral current density distribution in 6239 voxels (5 $\mathrm{mm}$ resolution), each voxel containing an equivalent current dipole. This 3dimensional solution space in which the inverse problem is solved is restricted to the cortical gray matter (and hippocampus). The head model for the inverse solution uses the electric potential lead field computed with a boundary element method applied to the MNI152 template (Fuchs, Kastner, Wagner, Hawes, \& Ebersole, 2002). Scalp electrode coordinates on the MNI brain are derived from the international 5\% system (Jurcak, Tsuzuki, \& Dan, 2007). The calculation of all reconstruction parameters was based on the computed common average reference. sLORETA units were scaled to amperes per square meter $\left(\mathrm{A} / \mathrm{m}^{2}\right)$.

\section{Results}

\section{Behavioral results}

As previously reported in Vocat et al. (2008), this task was successful in inducing a high number of errors for all participants (mean: $41.5 \%+/-13.3 \%$, min: $20.8 \%$, max: $65.8 \%$ ), whereas no single omission (lack of overt response during Go trials) was observed. Almost all errors $(99.7 \%)$ were verbally reported. These results confirmed that the implemented time pressure manipulation did not alter the perceived accuracy for performance. During the course of the experiment, the time pressure invoked by the feedback was efficient, since participants made systematically faster decisions $(\mathrm{p}<.001)$ after the calibration blocks (see Methods). Mean RT (computed from the onset of the imperative visual stimulus, the changing arrow) was $249+/-24 \mathrm{~ms}$ for Fast Hits, $323+/-22 \mathrm{~ms}$ for Slow Hits, and 249 +/-18 ms for Errors. Statistical comparisons (paired t-tests) showed that RTs were significantly slower for Slow Hits than either Fast hits $[\mathrm{t}(15)=19.12, \mathrm{p}<.001]$ or Errors $[\mathrm{t}(15)=20.40, \mathrm{p}<.001]$. Importantly, no significant difference was found between Fast Hits and Errors $[\mathrm{t}(15)=.15$, $\mathrm{p}=.88$ ], indicating that commission errors were not caused by slower perceptual decisions (or by lapses of either attention or readiness/arousal). An additional analysis was also performed 
to ascertain that errors did not vary as a function of the delay (randomly varying between 1000 and $2000 \mathrm{~ms}$ ) between the first black arrow (cue) and the changing arrow (target; see Supplementary Fig. 1A). Likewise, another additional control analysis was performed to look at the RT distribution for errors, relative to fast hits. Results of this analysis showed a tight overlap between these two RT distributions, confirming a similar speed for these two conditions (see Supplementary Fig. 1B). Therefore, this task enabled to compare these two opposite accuracy conditions (Fast hits vs. Errors), while the actual motor behavior (i.e., a simple motor key press) and behavioral speed (RT) were almost identical between these two opposite conditions.

\section{ERP results}

(1) The non-parametric statistical comparison between Fast hits and Errors did not reveal any significant GFP difference (Figs. 1A and 2A), suggesting that the strength (amplitude) of the ERP signal was comparable during this $500 \mathrm{~ms}$ pre-response time-period for these two conditions. As expected, because the putative motor preparation stage was visibly taking place earlier relative to motor response for Slow hits (Fig. 3A) compared to the two other experimental conditions (Fast Hits, Fig. 1A, and Errors, Fig. 2A), a significant GFP difference $(\mathrm{p}<.01)$ was found between Slow hits and Fast hits (from $172 \mathrm{~ms}$ to $96 \mathrm{~ms}$ before the onset of the response), as well as between Slow hits and Errors (from $140 \mathrm{~ms}$ to $118 \mathrm{~ms}$ before the onset of the response). In each case, this GFP difference indicated a significantly earlier increase of the strength of the signal during the pre-response baseline (relative to the onset of the response) for Slow hits (GFP peak: $105 \mathrm{~ms}$ before the response, Fig. 3A) compared to either the Fast hits (GFP peak: $48 \mathrm{~ms}$ before the response, Fig. 1A) or Errors (GFP peak: $50 \mathrm{~ms}$ before the response, Fig. 2A). Thus, Slow hits generated an earlier increase of the ERP signal in the pre-response time period (relative to response onset), as compared with Fast hits and Errors that each also led to a reliable power increase during the preresponse baseline (corresponding to the putative motor preparation stage), but with this amplitude increase occurring closer to response onset in these two latter conditions.

(2) I next tested whether Fast hits might differ from Errors when considering changes in the electric field configuration, which may occur irrespective of changes in strength (see methods). This was achieved by computing the global dissimilarity index (Lehmann \& Skrandies, 1980) and by subsequently comparing this index across conditions using randomization tests. In the Fast hits condition, I clearly found a single sharp increase of dissimilarity ( $\mathrm{p}<.01$ ), peaking $168 \mathrm{~ms}$ before the response (Fig. 1B). This result contrasted with the weaker topographic transition found for example between the ERN and Pe component (post-response). However, the electric field distributions of the ERN and Pe usually share some common geometric features (with a broad positive activity over centroposterior leads; see also Vocat et al., 2008 for a thorough presentation of the topographic transition between ERN and Pe) which may explain this difference between pre and postresponse dissimilarity changes. This unique and abrupt change of dissimilarity clearly came before the putative motor preparation stage, as reflected by the reliable power (GFP) increase 
(peaking $50 \mathrm{~ms}$ before the onset of the response) and "P component" occurring closer to motor response (RT) in this condition (Fig. 1A). This phasic increase of the dissimilarity unambiguously indicated that a reliable change of topography (and by extension functional microstate) occurred during the pre-response time period for Fast hits. By contrast, no similar single abrupt change of topographic dissimilarity could be found for Errors (Fig. 2B). I failed to identify a single and reliable increase of dissimilarity for Errors during the $500 \mathrm{~ms}$ preresponse time-period. Instead, changes in dissimilarity were clearly manifold $(p<.01)$ and thus less systematic during the $500 \mathrm{~ms}$ pre-response time-period (without any clear distinctive dissimilarity peak, Fig. 2B), compared to Fast hits (Fig. 1B). This was confirmed by a direct non-parametric statistical comparison, which confirmed a significant change of dissimilarity $(\mathrm{p}<.05)$ for Fast hits relative to Errors from $146 \mathrm{~ms}$ until $124 \mathrm{~ms}$ before the onset of the response (Fig. 1B), thus during a prolonged time-period that was immediately consecutive to the reliable dissimilarity increase found for Fast hits.

A supplementary analysis confirmed that this lack of dominant topographic change during the pre-response interval for errors was not simply due to a poorer signal to noise ratio (SNR) in this condition, relative to the fast or slow hits conditions (where more sweeps were included in the averages). Because fast hits were twice more frequent than errors, I used an odd-even average of the individual trials to compute new ERP waveforms (fast hits) containing the same number of trials (relative to errors). Then, dissimilarity was calculated again for this condition (fast hits, see Fig. 1D). Although the dissimilarity signal was visibly noisier (as could be anticipated when reducing trial number), a very similar pattern (including a main topographic transition during the pre-response interval) was nevertheless obtained for this new average (i.e. fast hits matched with errors for the number of trials included in the averages, see Fig. 1D), relative to Fig. 1B (all fast hits). Critically, the results of this supplementary analysis were still different, compared to the results obtained for errors, where no such main topographic transition (dissimilarity peak) was evidenced (see Fig. 2B). Hence, this new analysis enabled to rule out a poorer SNR for errors, relative to hits, that would account for the dissimilarity pattern reported in Fig. 2B.

Interestingly, a similar sharp increase of dissimilarity, unequivocally reflecting a topographic change (Lehmann \& Skrandies, 1980), could also be detected for Slow hits during the pre-response time period, though this abrupt change of dissimilarity clearly took place earlier for Slow hits (dissimilarity peak: $220 \mathrm{~ms}$ before the response, Fig. 3B) than Fast hits (dissimilarity peak: $168 \mathrm{~ms}$ before the response, Fig. 1B), while such dissimilarity peak was not evidenced for Errors (Fig. 2B). For Slow hits alike, this sharp increase of dissimilarity clearly preceded a subsequent GFP increase (GFP peak: $105 \mathrm{~ms}$ before the response, Fig. 3B), thought to index a motor preparation stage (Fig. 3A), thus providing a replication of the results already obtained for Fast hits (Figs 1A and 1B). A direct statistical comparison of topographic dissimilarity between Slow hits and Fast hits confirmed that changes in dissimilarity were significantly different $(\mathrm{p}<.05)$ during the two time periods in the preresponse baseline (220 ms and $168 \mathrm{~ms}$ before response onset), showing an earlier change (increase) of dissimilarity for Slow hits than Fast hits (220 ms before response, Fig. 3B), with a reversed effect during a later time period (168 ms before response, Fig. 1B). Finally, the 
comparison between Slow hits and Errors also revealed a significant difference $(p<.05) 220$ $\mathrm{ms}$ before motor response, indicated by a larger dissimilarity index for Slow hits than Errors (Figs. 2B and 3B). Altogether, these topographic dissimilarity results showed that a main topographic change occurred both for Fast hits (Fig. 1B) and Slow hits (Fig. 3B) during the pre-response time period (with this change occurring earlier for Slow hits than Fast hits), while a similar main alteration of the electric field configuration could not be detected for Errors (Fig. 2B).

(3) Next, I used a spatial cluster analysis (based on the K-means algorithm, see R.D. Pascual-Marqui et al., 1995) to better characterize for each condition (Fast hits, Slow hits and Errors) the exact sequence and distribution of electric field configurations during the $500 \mathrm{~ms}$ pre-response time period (Figs 1C, 2C and 3C). This analysis was primarily performed to determine whether the main topographic change identified by the previous dissimilarity analysis both for Fast hits and Slow hits was actually comparable (despite an obvious latency shift), as well as to further explore how errors might differ from these two other (correct) conditions in terms of sequence of electric field configurations during the $500 \mathrm{~ms}$ preresponse time period. The spatial cluster analysis (R.D. Pascual-Marqui et al., 1995) disclosed that the grand average ERP data during the $500 \mathrm{~ms}$ pre-response time period for the three conditions concurrently (Fast hits, Slow hits and Errors) could be reliably modeled by a solution with 3 different topographic maps, explaining 93\% of the variance. This solution revealed that the first topographic map was shared between the three conditions (Figs 1C, 2C and $3 \mathrm{C}$ ), ruling out the possibility that the initial topographic baseline activity would be already different for Errors (Fig. 2C), compared to either Fast (Fig. 1C) or Slow hits (Fig. 3C). However, following this initial (baseline) map shared across the three experimental conditions, a clear topographic difference was evidenced between experimental conditions. Whereas a specific topographic map was found to suddenly replace the initial topographic map both for Fast and Slow hits (Figs. 1C and 3C), this second distinctive topographic map was basically suppressed at the group level (grand average ERP data) for Errors (Fig. 2C). Remarkably, for Fast and Slow hits, the topographic transition precisely occurred during the time period when a phasic increase of dissimilarity was previously evidenced (see point 2 here above; $220 \mathrm{~ms}$ and $168 \mathrm{~ms}$ before response onset for Slow and Fast hits, respectively), corroborating the statistical outcome of the analysis of topographic dissimilarity. This cluster analysis therefore confirmed that a reliable topographic transition (and therefore a change of the underlying neural generators, see Lehmann \& Skrandies, 1980) occurred both for Fast and Slow hits at a precise moment during the pre-response time period (being earlier for Slow than Fast hits, see Figs. 1C and 3C), whereas this sudden change of the electric field configuration was markedly reduced for Errors (Fig. 2C). These differences were verified by statistical tests performed on the GEV values (Fig. 5A) that were extracted by fitting these dominant topographic maps (identified at the group level, grand average ERP results) to individual ERP data for each participant $(n=16)$ in each condition $(n=3)$ (see Lehmann \& Skrandies, 1980 C.M. Michel et al., 1999; Murray et al., 2008; Pourtois et al., 2008). For this purpose, I fitted these two topographic maps (i.e., the initial baseline map, later followed by a different transition map for Fast and Slow hits) back to the individual ERP data during a long time-interval encompassing the putative topographic transition in all three conditions (i.e., 
from 210 to $90 \mathrm{~ms}$ before motor response), as objectively (and independently) determined by the analysis of dissimilarity (see point 2). Please note that there was a reliable shift for the main topographic transition between fast (150-100 ms pre-response) and slow hits (220-150 ms pre-response), which thus accounted for the use of this specific interval (i.e., from 210 to $90 \mathrm{~ms}$ before motor response) for the back-fitting. Hence, the selection of this time period or interval (for the back-fitting) was not arbitrary, but directly motivated by the outcome of the spatial cluster and dissimilarity analyses, following standard practice (see Murray et al., 2008). I extracted the goodness of fit (or GEV) of each of these two topographic maps in this time interval for each subject $(n=16)$ and each condition $(n=3)$, and submitted these values to a 2(Map) x 3(Condition) repeated measures Analysis of Variance (ANOVA). This ANOVA (Fig. 5A) verified a highly significant Map x Condition interaction $[F(2,30)=27.58, p<.001]$. Whereas the explained variance of these two maps was low and similar for Errors $[\mathrm{t}(15)=.25$, $\mathrm{p}=.81$ ], the explained variance of the second (transition) map was substantially larger than the first map during this time interval (consistent with a reliable topographic transition), both for Fast hits $[\mathrm{t}(15)=3.20, \mathrm{p}=.006]$ and Slow hits $[\mathrm{t}(15)=9.82, \mathrm{p}<.001$, see Fig. 4A]. Importantly, the critical transition map had a significantly larger variance for Slow hits than Errors $[\mathrm{t}(15)=$ 7.79, $\mathrm{p}<.001]$, and for Fast hits than Errors $[\mathrm{t}(15)=3.34, \mathrm{p}=.004$, see Fig. 4A], corroborating the assumption of a genuine topographic change for Slow and Fast hits, relative to Errors where a similar topographic change was not observed.

I also extracted the duration of these two maps during this time interval (i.e., from 210 to $90 \mathrm{~ms}$ before motor response), and again submitted these values to a similar 2(Map) $\mathrm{x}$ 3(Condition) repeated measures ANOVA. This analysis also disclosed a highly significant Map x Condition interaction $[\mathrm{F}(2,30)=25.52, \mathrm{p}<.001]$, indicating a substantially longer duration of the transition map for Slow hits compared to Errors [t $(15)=7.20, p<.001]$, and for Fast hits compared to Errors $[\mathrm{t}(15)=2.22, \mathrm{p}=.042]$. By contrast, the initial (baseline) map had a longer duration for Errors, relative to either Slow Hits $[\mathrm{t}(15)=7.30, \mathrm{p}<.001]$ or Fast hits $[\mathrm{t}(15)=2.20, \mathrm{p}=.043]$ during this specified pre-response time interval. Altogether, these statistical results lent support to the assumption that a main topographic transition took place during the pre-response time period, equally so for Slow hits and Fast hits (though at an earlier time for Slow hits than Fast hits), whereas this topographic transition was markedly reduced for Errors (Fig. 5A). Importantly, these topographic analyses converged with the statistical analyses of dissimilarity (see point 2 here above) and showed that a main topographic transition was merely absent for Errors, relative to the two other correct conditions (Fig. 5A). Therefore, these results were intriguing at first sight, as they suggested that Errors could be partly explained by ERP topographic changes during the pre-response time-period (i.e., from 210 to $90 \mathrm{~ms}$ before motor response), hence when the erroneous key presses (false alarms) had not been made yet, and presumably the participants were still processing the visual stimuli (colored arrow) during this time period. Moreover, because the main motor preparation stage was found to be similar between all three conditions (Figs. 1A, $2 \mathrm{~A}$ and $3 \mathrm{~A}$ ), these results suggested that a differential neural event, clearly preceding motor preparation, actually differentiated Fast and Slow hits from Errors (Figs. 1B, 2B and 3B). 
I also run several control analyses to establish whether this topographic change identified prior to the response for fast and slow hits could actually be explained by differential stimulus-locked potentials. Accordingly, I performed new statistical analyses taking as reference point the onset of the changing stimulus, rather than the response (see Fig. 4). As can be seen from Fig. 4, these analyses clearly failed to disclose any obvious topographic difference between hits (either fast or slow) and errors during the $250 \mathrm{~ms}$ time interval following the onset of the visual stimulus (color arrow) and encompassing the early visual ERPs, including the N1. Because the task was initially designed in such a way to minimize low-level differences between go and nogo trials, this result was actually not surprising. Noteworthy, $250 \mathrm{~ms}$ post-stimulus onset was used as an objective time limit in these auxiliary analyses since mean RT was precisely $250 \mathrm{~ms}$, both for errors and fast hits (see behavioral results). However, the stimulus-locked analyses (see Fig. 4A) confirmed that errors were unambiguously generating an ERN component peaking $\sim 300 \mathrm{~ms}$ after stimulus onset (see Falkenstein et al., 2000), whereas successful inhibitions on nogo trials (i.e. correct rejections) generated a clear fronto-central nogo P3 component $\sim 370$ ms post-stimulus onset (Kok, 1986; Nieuwenhuis, Yeung, van den Wildenberg, \& Ridderinkhof, 2003). I next performed an extra spatial cluster analysis using the $500 \mathrm{~ms}$ time interval following stimulus onset (Fig. 4B and 4C) to assess whether errors might differ from hits during early stages of stimulus processing, hence during a time interval likely overlapping with the topographic change identified by the previous response-locked ERP analyses. A solution with 7 dominant topographic maps was found to explain $94 \%$ of the variance (Fig. 4C). Importantly, no reliable topographic difference between conditions (hits vs. errors) could be evidenced during the initial $250 \mathrm{~ms}$ time interval following the onset of the visual stimulus, confirming that the large topographic change found during the pre-response time interval for fast and slow hits (and being markedly reduced for errors, see Figs. 1-3) was not confounded by a systematic change across conditions related to early stimulus processing. After $250 \mathrm{~ms}$ post-stimulus onset (and hence after the manual response), this analysis confirmed reliable topographic differences between conditions (Fig. 4B), as previously described (see Vocat et al., 2008).

In order to more directly relate this reduction of topographic transition during the preresponse baseline with brain mechanisms of error detection, I next performed a standard correlation analysis to assess whether early error detection, as formally defined by the magnitude of the ERN component (see Falkenstein et al., 2000), was linked to this earlier reduced topographic transition occurring during the pre-response time period (Fig. 5B). I therefore performed a Pearson correlation across the 16 participants between the size of the ERN component following errors (as measured at the reference electrode $\mathrm{FCz}$, see Vocat et al., 2008) and the duration of this transition topographic map (computed during the same time interval used for the topographic analyses, namely from 210 to $90 \mathrm{~ms}$ before motor response) in this condition (Errors). Note that although the previous analysis suggested that the main topographic transition during the pre-response interval was merely suppressed at the group level for errors (see Fig. 2C), relative to hits, there was nevertheless some variation across participants in the expression of this transition map in this condition (errors; see Fig. 5A). Moreover, this correlation analysis was carried out using the duration rather than the GEV of the dominant map, as the former (but not the latter) measure turned out to reliably predict the 
magnitude of the ERN component, although similar results were obtained for these two measures (duration and GEV) when back fitting these dominant maps (see here above). Remarkably, this analysis (Fig. 5B) showed a negative correlation between the magnitude of the ERN and the duration of the transition topographic map during this pre-specified preresponse time period $[\mathrm{r}(16)=-.52, \mathrm{p}=.041 ; \mathrm{r}(15)=-.70, \mathrm{p}=.004$ after removing the data of one outlier]. Across participants, the larger the duration of this transition map (which was, overall, reduced for errors relative to correct hits, as described above), the lower the amplitude of the ERN component, suggesting that early error detection mechanisms (as reflected by the size of the ERN) were influenced by this preparatory ERP activity occurring 190ms earlier during the pre-response time-period (Fig. 5B). Early error detection (ERN) was therefore enhanced when this preceding transition topographic map had a shorter duration, corroborating the assumption that the ERP activity taking place during the pre-response time period was somehow contributing to brain mechanisms of error detection. These results suggest a functional link between two distant and non-overlapping neural events during action monitoring, the former taking place $\sim 150 \mathrm{~ms}$ before the motor response and the latter immediately after this response (see Fig. 2A). By comparison, the correlation between the duration of this transition map during the pre-response time period and the amplitude of the Pe component following errors (see Vocat et al., 2008) was not significant ( $p>.05$ ), suggesting a component specific effect.

(4) Finally, I used sLORETA to gain insight into the putative neural generators of these two topographic maps (Fig. 5), focusing on the initial (baseline) and the transition topographic maps; the duration of the latter neural event accounting for some of the amplitude variance at the level of the ERN component (Fig. 5B). Whereas the neural generators of the initial (baseline) map were primarily localized within medial regions of the occipital cortex (Brodmann Area 19; Cuneus; right: $+5 x,-90 y,+30 z$; left: $-4 x,-92 y,+24 z$, see Fig. 6A), consistent with the early sensory processing of the imperative visual stimulus in this task, the brain sources of the transition map were found mainly in more dorsal cortical regions, at the border between the cuneus and precuneus, in the parietal cortex (Brodmann Area 7; right: 10x, $-80 y,+43 z$; left: $-8 x,-80 y,+40 z$, see Fig. 6B). Using sLORETA, I next performed a direct statistical comparison (paired t-test) between Fast Hits and Errors in this inverse solution space (Fig. 6C), during the pre-response time period where the main topographic transition was found to take place for Fast hits, while being markedly reduced for Errors (170$150 \mathrm{~ms}$ before response, see points 1 and 2 here above). I performed the statistical comparison in the inverse solution space during this specific time period because it corresponded to the interval when the topographic change was the most obvious (and significant) for fast hits (170-150 ms pre-response, see Fig. 1B). When making this interval larger (210-90 ms preresponse, see results of the spatial cluster analysis here above), the outcome for the inverse solution remained similar (see Fig. 6C) but the statistical values reliably decreased. This statistical comparison revealed a highly significant $(\mathrm{p}<.001)$ activation for Fast hits relative to Errors, circumscribed within the posterior parietal cortex during this time interval (Fig. 6C). Maxima were found in the superior parietal lobule/precuneus (right: $5 \mathrm{x},-70 \mathrm{y},+55 \mathrm{z}, \mathrm{t}$-value: 5.92, $\mathrm{p}<.001$; left: $-5 \mathrm{x},-70 \mathrm{y},+55 \mathrm{z}$, t-value: 5.50, $\mathrm{p}<.001$, see Fig. 6C). Therefore, this statistical comparison suggested that the neural generators underlying the main topographic 
transition occurring for Fast hits relative to Errors during the pre-response time period primarily implicated regions of the posterior parietal cortex (precuneus). By comparison, the neural generators of the ERN topographic scalp map were mainly localized, as expected, within the medial frontal gyrus (right: $+5 \mathrm{x},-22 \mathrm{y},+57 \mathrm{z}$; left: $-4 \mathrm{x},-22 \mathrm{y},+55 \mathrm{z}$ ), extending more ventrally towards the rostral cingulate gyrus (right: $+9 x,-17 y,+45 z$; left: $-9 x,-17 y,+45 z$, see Fig. 6D), consistent with earlier source localization results for the ERN component (see Dehaene et al., 1994; Herrmann, Rommler, Ehlis, Heidrich, \& Fallgatter, 2004; Debener et al., 2005; Pizzagalli et al., 2006; van Veen \& Carter, 2006; O'Connell et al., 2007; Vocat et al., 2008).

\section{Discussion}

In this study, I analyzed pre-response ERP data recorded during a new speeded go/nogo task (see Vocat et al., 2008) and compared two opposite accuracy conditions, namely Fast (correct) hits vs. Errors (false alarms on nogo trials), while the behavioral speed (RT) was similar between these two conditions, ruling out the possibility that commission errors were simply occurring in this task because participants had overall lower vigilance or reactivity during these incorrect responses. I tested the hypothesis that errors (unavoidable false alarms) might already differ from correct hits during the pre-response preparatory time period, in keeping with previous cognitive control studies that have identified higher-order proactive attentional changes occurring during the pre-stimulus (or pre-response) time period under increased task demands (see Braver et al., 2007; Braver et al., 2009), such as during the inhibition of a prepotent response (as typically required by go/nogo tasks, as used here in this study), or during task shifting (see Bunge et al., 2002; Barber \& Carter, 2005; Robbins, 2007; see also Gevins et al., 1987; Padilla et al., 2006; Li et al., 2007). Thus, under high attentional demands, top-down attentional control effects in the posterior parietal lobe (including regions of the precuneus) were found to take place before the onset of the imperative stimulus or response (see Barber \& Carter, 2005; Li et al., 2007 for recent fMRI evidence), consistent with the assumption that cognitive control involves a network of frontal and parietal brain regions, some of which may generate an early anticipatory top-down attentional signal to help and guide the actual selection of S-R associations (Rushworth et al., 2001; Bunge et al., 2002; Rushworth \& Taylor, 2006; Robbins, 2007; see also Corbetta \& Shulman, 2002; Weissman et al., 2006; Orr \& Weissman, 2009). The goal of this study was to further explore, using advanced topographic analyses of scalp ERP data, the electrophysiological correlates of these putative "anticipatory" attentional effects that may foreshadow response errors.

These new analyses confirmed that correct responses (either Fast or Slow hits) were associated with a distinctive neural event during the pre-response time period, which was, however, markedly reduced for Errors. Whereas this topographic modification occurred earlier for Slow than for Fast hits, it was substantially decreased for Errors. Moreover, I could confirm that this topographic change was actually the same for Slow and Fast correct hits (similar topographic transition effect), despite this clear latency shift across these two accurate conditions, suggesting that this distinctive neural event was related to cognitive or attentional 
control, rather than to either motor preparation or speed per se. I showed, using topographic mapping analyses, that this neural event corresponded to a sharp and main topographic transition, reflecting a genuine change of functional microstates (see Lehmann \& Skrandies, 1980; C.M. Michel et al., 1999). More precisely, I found that for Fast and Slow hits, unlike Errors, an initial baseline topographic activity (which was common to all three conditions) underwent an abrupt configuration change during the pre-response time period, swiftly moving from an occipital (baseline) to a posterior parietal (transition) microstate (implicating a region of the precuneus), as revealed by the distributed source localization results, and statistical analyses. Importantly, control analyses looking at stimulus-locked effects failed to disclose any reliable change early on following stimulus onset that could potentially account for the pre-response topographic change found in this study. These control analyses therefore confirmed that this pre-response topographic change, which was markedly reduced for errors relative to hits, was related to action monitoring processes rather than stimulus encoding processes.

Source localization results based on sLORETA (R. D. Pascual-Marqui, 2002) showed that a circumscribed activation within the precuneus differentiated Fast hits from Errors, during the time-interval precisely corresponding to the topographic transition for Fast hits, but not Errors (170-150 ms before response). Previous fMRI studies have already suggested that the precuneus (superior parietal lobule) plays a critical role in voluntarily (endogenously) shifting (directing) attentional resources towards the relevant stimulus (or S-R associations) properties (see Rushworth et al., 2001; Corbetta \& Shulman, 2002). Among its hypothesized functional roles, this posterior parietal region would contribute to readying the cognitive system for task performance under high attentional demands, including during the inhibition of a prepotent response tendency (see Barber \& Carter, 2005). Alternatively, this posterior parietal cortex region could also play a role in attentional mechanisms of response selection (in concert with the DLPFC, see also Botvinick et al., 2001; Ridderinkhof et al., 2004), by pre-activating and perhaps narrowing the repertoire of potential S-R associations (see Bunge et al., 2002). Finally, the critical contribution of the posterior parietal cortex could be to reupdate representations for attention (Rushworth \& Taylor, 2006). Here I found a similar precuneus activation for Fast hits (and Slow hits as well), but not for Errors, occurring during the pre-response anticipatory time period, when participants presumably processed the imperative visual stimulus (a colored green arrow) and were asked to timely select the appropriate motor response (i.e., to perform a rapid key press in response to this go signal). This precuneus activation could therefore reflect a top-down attentional shift during the preresponse time period, meant to enhance the correct S-R association (Bunge et al., 2002), or alternatively to break up the ongoing sensory processing of the imperative visual stimulus and orient towards the motor preparation stage (Corbetta \& Shulman, 2002; Woldorff et al., 2004). Noteworthy was the absence of this distinctive precuneus activation characteristic of Errors, consistent with the early contribution of this posterior parietal cortex region in efficiently directing attentional resources towards the relevant S-R association (see Barber \& Carter, 2005). 
The statistical analyses also confirmed that this pre-response ERP effect truly corresponded to a change of the electric field configuration (as reflected by the map dissimilarity index, see Lehmann \& Skrandies, 1980), as opposed to a change of strength (as reflected by the GFP, see Lehmann \& Skrandies, 1980), again arguing against a simple explanation in terms of impaired arousal, vigilance or overall decreased motor preparation during the pre-response time period eventually leading to Errors. In fact, the putative motor preparation stage was found to be similar across the three conditions (as was also the case for the first initial topographic activity involving medial occipital regions), and this topographic transition effect clearly took place before this motor preparation stage (see Figs. 1A and 1B). This change of the electric field configuration is indicative of changes in the underlying generator configuration (see Lehmann, 1987), as further verified by the source localization results, which clearly identified a shift in the distribution of neural generators from occipital to posterior parietal (precuneus) regions. I also used a spatial cluster analysis (R.D. PascualMarqui et al., 1995) to better characterize, in each condition, the expression and sequence of topographic changes during the $500 \mathrm{~ms}$ pre-response time period. These additional analyses confirmed that Errors reliably differed from either Fast or Slow hits during this time period. These topographic mapping results showed that the precuneus transition map was not expressed to the same extent at the group level for Errors, relative to the two other (correct) conditions, in agreement with the statistical outcome of the topographic dissimilarity analysis (Lehmann \& Skrandies, 1980). Moreover, I found that across participants, this precuneus transition map accounted for some of the amplitude variance of the response-related ERN component for errors (Falkenstein et al., 2000), which peaked $190 \mathrm{~ms}$ later and was therefore not adjacent to this early topographic alteration during the pre-response time-period, and clearly involved rostral cingulate regions (Fig. 5D). These two neural events were separated from one another by several other intervening neural events, including motor preparation. The source localization results confirmed that the neural generators of the ERN involved regions of the rostral cingulate gyrus (see Debener et al., 2005; Vocat et al., 2008). A correlation analysis revealed that the longer this transition map, the smaller the amplitude of the ERN component, corroborating the assumption that this early topographic ERP effect during the pre-response time period also somehow participated in early error detection processes. Furthermore, these results showed that this correlation was specific to the ERN component, as the duration of the transition map did not correlate with the amplitude of the error-related Pe component (Falkenstein et al., 2000; O'Connell et al., 2007), which immediately followed the ERN component (see Vocat et al., 2008). Altogether, these new results are therefore compatible with the notion that the rostral cingulate cortex is interconnected with more posterior parietal regions involved in top-down attentional control and that higher-order attentional deficits may also therefore influence cognitive control mechanisms within the dACC (see also Li et al., 2008; Orr \& Weissman, 2009).

Although the ERN component was previously shown to be primarily time-locked and, to a lesser degree, phased-locked to the subject's motor response ("response-triggered component", see Coles et al., 2001; Luu et al., 2004), these new results suggest that the amplitude of this early error-related component was also partly determined by systematic attentional changes in the posterior parietal cortex (precuneus) occurring during the pre- 
response (baseline) time period, roughly 150 milliseconds before the response took place ("anticipatory" component). These findings therefore suggest that the size of the ERN was not exclusively determined by an online matching process between the expected and the actual motor response (implicating primarily regions of the dorsal ACC or medial frontal cortex, see Falkenstein et al., 1991; Scheffers et al., 1996; Coles et al., 2001; Nieuwenhuis et al., 2001), but that this early error detection mechanism was also somehow influenced by the duration (and presumably efficiency) of a preceding and systematic attentional control process, primarily implicating regions of the precuneus (see Barber \& Carter, 2005; Margulies et al., 2007). These new results showed that the magnitude of the early error detection process (as reflected by the ERN component) could be partly predicted by top-down attentional changes occurring within the posterior parietal cortex during the pre-response time period, emphasizing the distinctive contributions of medial frontal, as opposed to posterior parietal regions during action monitoring (see Bunge et al., 2002). Hence, the perception of errors (or conflicts) do not only rapidly increase attentional resources and cognitive control effects through a dynamic interplay between the dACC and DLPFC (see Botvinick et al., 2001), but under some circumstances (e.g., when the task demands require a high level of attentional control, as in the present case, see Vocat et al., 2008), an anticipatory (top-down) attentional component generated in the precuneus may also have a proactive impact on regions of the dACC, selectively involved in error (or conflict) monitoring (see also Rushworth et al., 2001; Braver et al., 2007; Braver et al., 2009)). These new results have implications for cognitive models of the ERN component (Scheffers et al., 1996; Holroyd \& Coles, 2002; Botvinick et al., 2001), which typically do not weight action monitoring or action regulation functions with the potential contribution of higher-order anticipatory attentional factors (but see Yeung et al., 2004). These results showed that the ERN component, reflecting either conflict detection (Botvinick et al., 2001; Yeung et al., 2004) or reinforcement learning (Holroyd \& Coles, 2002), may be enhanced by the reduction (i.e., shorter duration) of an earlier non-adjacent anticipatory attentional effect taking place in the precuneus $\sim 190 \mathrm{~ms}$ earlier. Attentional shifts during the preparatory baseline time period were already shown to influence the sensory processing of (upcoming/imminent) visual stimuli (see Kondakor et al., 1995; Kastner, Pinsk, De Weerd, Desimone, \& Ungerleider, 1999; Super, van der Togt, Spekreijse, \& Lamme, 2003; Pourtois et al., 2006). Here I described a similar effect for action monitoring, where the early detection of errors (as reflected by the ERN component) was found to be influenced by the extent to which a putative anticipatory attentional control process, involving posterior parietal brain regions (precuneus), was expressed during the pre-response time period (see also Gevins et al., 1987; Weissman et al., 2006). As such, these new ERP results shed new light on the interaction effects between attention and decision making mechanisms in the human brain. Finally, these new findings also illustrate the added value of alternative topographic ERP mapping techniques (see Pourtois et al., 2008; Murray et al., 2008) to gain insight into the precise spatio-temporal dynamics of cognitive control and action monitoring processes in the human brain, relative to more traditional peak analyses (Picton et al., 2000). 


\section{Acknowledgements}

This work is supported by grants from the European Research Council (Starting Grant \#200758) and Ghent University (BOF Grant \#05Z01708). Thanks to Dr. Roland Vocat for earlier discussions on error detection brain mechanisms, and to Dr. Monica Dhar for her comments on an earlier draft of this manuscript.

\section{References}

Allain, S., Carbonnell, L., Falkenstein, M., Burle, B., \& Vidal, F. (2004). The modulation of the Ne-like wave on correct responses foreshadows errors. Neurosci Lett, 372(1-2), 161-166.

Astafiev, S. V., Shulman, G. L., Stanley, C. M., Snyder, A. Z., Van Essen, D. C., \& Corbetta, M. (2003). Functional organization of human intraparietal and frontal cortex for attending, looking, and pointing. J Neurosci, 23(11), 4689-4699.

Barber, A. D., \& Carter, C. S. (2005). Cognitive control involved in overcoming prepotent response tendencies and switching between tasks. Cereb Cortex, 15(7), 899-912.

Botvinick, M. M., Braver, T. S., Barch, D. M., Carter, C. S., \& Cohen, J. D. (2001). Conflict monitoring and cognitive control. Psychol Rev, 108(3), 624-652.

Braver, T. S., Gray, J. R., \& Burgess, G. C. (2007). Explaining the many varieties of working memory variation: Dual mechanisms of cognitive control. In A. R. A. Conway, C. Jarrold, M. J. Kane, A. Miyake \& J. N. Towse (Eds.), Variation in Working Memory (pp. 76-106). Oxford: Oxford University Press.

Braver, T. S., Paxton, J. L., Locke, H. S., \& Barch, D. M. (2009). Flexible neural mechanisms of cognitive control within human prefrontal cortex. Proc Natl Acad Sci U S A, 106(18), 7351-7356.

Bunge, S. A., Hazeltine, E., Scanlon, M. D., Rosen, A. C., \& Gabrieli, J. D. (2002). Dissociable contributions of prefrontal and parietal cortices to response selection. Neuroimage, 17(3), 1562-1571.

Bush, G., Luu, P., \& Posner, M. I. (2000). Cognitive and emotional influences in anterior cingulate cortex. Trends in Cognitive Sciences, 4(6), 215-222.

Carter, C. S., Botvinick, M. M., \& Cohen, J. D. (1999). The contribution of the anterior cingulate cortex to executive processes in cognition. Rev Neurosci, 10(1), 49-57.

Carter, C. S., Braver, T. S., Barch, D. M., Botvinick, M. M., Noll, D., \& Cohen, J. D. (1998). Anterior cingulate cortex, error detection, and the online monitoring of performance. Science, 280(5364), 747-749.

Cohen, J. D., Perlstein, W. M., Braver, T. S., Nystrom, L. E., Noll, D. C., Jonides, J., et al. (1997). Temporal dynamics of brain activation during a working memory task. Nature, 386(6625), 604-608.

Coles, M. G., Gratton, G., \& Donchin, E. (1988). Detecting early communication: using measures of movement-related potentials to illuminate human information processing. Biol Psychol, 26(1-3), 69-89.

Coles, M. G., Scheffers, M. K., \& Holroyd, C. B. (2001). Why is there an ERN/Ne on correct trials? Response representations, stimulus-related components, and the theory of errorprocessing. Biol Psychol, 56(3), 173-189.

Corbetta, M., \& Shulman, G. L. (2002). Control of goal-directed and stimulus-driven attention in the brain. Nat Rev Neurosci, 3(3), 201-215. 
Debener, S., Ullsperger, M., Siegel, M., Fiehler, K., von Cramon, D. Y., \& Engel, A. K. (2005). Trial-by-trial coupling of concurrent electroencephalogram and functional magnetic resonance imaging identifies the dynamics of performance monitoring. Journal of Neuroscience, 25(50), 11730-11737.

Dehaene, S., Posner, M. I., \& Tucker, D. M. (1994). Localization of a neural system for error detection and compensation. Psychological Science, 5(5), 303-305.

Dempster, F. N., \& Corkill, A. J. (1999). Interference and inhibition in cognition and behavior: Unifying themes for educational psychology. Educational Psychology Review, 11(1), 1-88.

Desimone, R., \& Duncan, J. (1995). Neural mechanisms of selective visual attention. Annu Rev Neurosci, 18, 193-222.

Dreher, J. C., \& Berman, K. F. (2002). Fractionating the neural substrate of cognitive control processes. Proc Natl Acad Sci U S A, 99(22), 14595-14600.

Eichele, T., Debener, S., Calhoun, V. D., Specht, K., Engel, A. K., Hugdahl, K., et al. (2008). Prediction of human errors by maladaptive changes in event-related brain networks. Proc Natl Acad Sci U S A, 105(16), 6173-6178.

Falkenstein, M., Hohnsbein, J., Hoormann, J., \& Blanke, L. (1991). Effects of crossmodal divided attention on late ERP components. II. Error processing in choice reaction tasks. Electroencephalogr Clin Neurophysiol, 78(6), 447-455.

Falkenstein, M., Hoormann, J., Christ, S., \& Hohnsbein, J. (2000). ERP components on reaction errors and their functional significance: a tutorial. Biological Psychology, 51(2-3), 87-107.

Friedman, N. P., \& Miyake, A. (2004). The relations among inhibition and interference control functions: a latent-variable analysis. J Exp Psychol Gen, 133(1), 101-135.

Fuchs, M., Kastner, J., Wagner, M., Hawes, S., \& Ebersole, J. S. (2002). A standardized boundary element method volume conductor model. Clinical Neurophysiology 113, 702-712.

Gevins, A. S., Morgan, N. H., Bressler, S. L., Cutillo, B. A., White, R. M., Illes, J., et al. (1987). Human neuroelectric patterns predict performance accuracy. Science, 235(4788), 580-585.

Gratton, G., Coles, M. G., Sirevaag, E. J., Eriksen, C. W., \& Donchin, E. (1988). Pre- and poststimulus activation of response channels: a psychophysiological analysis. $J$ Exp Psychol Hum Percept Perform, 14(3), 331-344.

Gratton, G., Coles, M. G. H., \& Donchin, E. (1983). A New Method for Off-Line Removal of Ocular Artifact. Electroencephalography and Clinical Neurophysiology, 55(4), 468484.

Hajcak, G., Nieuwenhuis, S., Ridderinkhof, K. R., \& Simons, R. F. (2005). Error-preceding brain activity: robustness, temporal dynamics, and boundary conditions. Biol Psychol, 70(2), 67-78.

Herrmann, M. J., Rommler, J., Ehlis, A. C., Heidrich, A., \& Fallgatter, A. J. (2004). Source localization (LORETA) of the error-related-negativity (ERN/Ne) and positivity (Pe). Brain Res Cogn Brain Res, 20(2), 294-299.

Holroyd, C. B., \& Coles, M. G. (2002). The neural basis of human error processing: reinforcement learning, dopamine, and the error-related negativity. Psychological Review, 109(4), 679-709.

Jurcak, V., Tsuzuki, D., \& Dan, I. (2007). 0/20, 10/10, and 10/5 systems revisited: Their validity as relative head-surface-based positioning systems. NeuroImage, 34, 16001611. 
Kastner, S., Pinsk, M. A., De Weerd, P., Desimone, R., \& Ungerleider, L. G. (1999). Increased activity in human visual cortex during directed attention in the absence of visual stimulation. Neuron, 22(4), 751-761.

Kastner, S., \& Ungerleider, L. G. (2000). Mechanisms of visual attention in the human cortex. Annu Rev Neurosci, 23, 315-341.

Koenig, T., \& Melie-Garcia, L. (2010). A Method to Determine the Presence of Averaged Event-Related Fields Using Randomization Tests. Brain topography.

Kok, A. (1986). Effects of degradation of visual stimulation on components of the eventrelated potential (ERP) in go/nogo reaction tasks. Biol Psychol, 23(1), 21-38.

Kondakor, I., Pascual-Marqui, R. D., Michel, C. M., \& Lehmann, D. (1995). Event-Related Potential Map Differences Depend on the Prestimulus Microstates. Journal of Medical Engineering \& Technology, 19(2-3), 66-69.

Lavie, N. (2005). Distracted and confused?: selective attention under load. Trends Cogn Sci, 9(2), 75-82.

Lehmann, D. (1987). Principles of spatial analysis. In A. S. Gevins \& D. Remond (Eds.), Handbook of electroencephalography and clinical neurophysiology. Methods of analysis of brain electrical and magnetic signals (pp. 309-354). Amsterdam: Elsevier.

Lehmann, D., \& Skrandies, W. (1980). Reference-free identification of components of checkerboard-evoked multichannel potential fields. Electroencephalogr Clin Neurophysiol, 48(6), 609-621.

Levy, R., \& Goldman-Rakic, P. S. (1999). Association of storage and processing functions in the dorsolateral prefrontal cortex of the nonhuman primate. J Neurosci, 19(12), 51495158 .

Li, C. S., Yan, P., Bergquist, K. L., \& Sinha, R. (2007). Greater activation of the "default" brain regions predicts stop signal errors. Neuroimage, 38(3), 640-648.

Li, C. S., Yan, P., Chao, H. H., Sinha, R., Paliwal, P., Constable, R. T., et al. (2008). Errorspecific medial cortical and subcortical activity during the stop signal task: a functional magnetic resonance imaging study. Neuroscience, 155(4), 1142-1151.

Luu, P., Tucker, D. M., \& Makeig, S. (2004). Frontal midline theta and the error-related negativity: neurophysiological mechanisms of action regulation. Clinical Neurophysiology, 115(8), 1821-1835.

MacDonald, A. W., Cohen, J. D., Stenger, V. A., \& Carter, C. S. (2000). Dissociating the role of the dorsolateral prefrontal and anterior cingulate cortex in cognitive control. Science, 288(5472), 1835-1838.

Manly, B. F. (1991). Randomization and Monte Carlo Methods in Biology. London, UK: Chapman \& Hall.

Margulies, D. S., Kelly, A. M., Uddin, L. Q., Biswal, B. B., Castellanos, F. X., \& Milham, M. P. (2007). Mapping the functional connectivity of anterior cingulate cortex. Neuroimage, 37(2), 579-588.

Mazaheri, A., Nieuwenhuis, I. L., van Dijk, H., \& Jensen, O. (2009). Prestimulus alpha and mu activity predicts failure to inhibit motor responses. Hum Brain Mapp, 30(6), 17911800 .

Mazziotta, J., Toga, A., Fox, P., Lancaster, J., Zilles, K., woods, R., et al. (2001). A probabilistic atlas and reference system for the human brain: International Consortium for Brain Mapping (ICBM). Philos. Trans. R. Soc. Lond. B. Biol. Sci., 356, 12931322.

Michel, C. M., Seeck, M., \& Landis, T. (1999). Spatiotemporal Dynamics of Human Cognition. News Physiological Science, 14, 206-214. 
Michel, C. M., Thut, G., Morand, S., Khateb, A., Pegna, A. J., Grave de Peralta, R., et al. (2001). Electric source imaging of human brain functions. Brain Res Brain Res Rev, 36(2-3), 108-118.

Murray, M. M., Brunet, D., \& Michel, C. M. (2008). Topographic ERP analyses: a step-bystep tutorial review. Brain Topogr, 20(4), 249-264.

Nieuwenhuis, S., Holroyd, C. B., Mol, N., \& Coles, M. G. (2004). Reinforcement-related brain potentials from medial frontal cortex: origins and functional significance. Neurosci Biobehav Rev, 28(4), 441-448.

Nieuwenhuis, S., Ridderinkhof, K. R., Blom, J., Band, G. P., \& Kok, A. (2001). Error-related brain potentials are differentially related to awareness of response errors: evidence from an antisaccade task. Psychophysiology, 38(5), 752-760.

Nieuwenhuis, S., Yeung, N., van den Wildenberg, W., \& Ridderinkhof, K. R. (2003). Electrophysiological correlates of anterior cingulate function in a go/no-go task: effects of response conflict and trial type frequency. Cogn Affect Behav Neurosci, $3(1), 17-26$.

O'Connell, R. G., Dockree, P. M., Bellgrove, M. A., Kelly, S. P., Hester, R., Garavan, H., et al. (2007). The role of cingulate cortex in the detection of errors with and without awareness: a high-density electrical mapping study. Eur J Neurosci, 25(8), 2571-2579.

Orr, J. M., \& Weissman, D. H. (2009). Anterior cingulate cortex makes 2 contributions to minimizing distraction. Cereb Cortex, 19(3), 703-711.

Padilla, M. L., Wood, R. A., Hale, L. A., \& Knight, R. T. (2006). Lapses in a prefrontalextrastriate preparatory attention network predict mistakes. J Cogn Neurosci, 18(9), $1477-1487$.

Pascual-Marqui, R. D. (2002). Standardized low-resolution brain electromagnetic tomography (sLORETA): technical details, Methods Find. Exp. Clin. Pharmacol. , 24D, 5-12.

Pascual-Marqui, R. D., Michel, C. M., \& Lehmann, D. (1995). Segmentation of brain electrical activity into microstates: model estimation and validation. IEEE Trans Biomed Eng, 42, 658-665.

Picton, T. W., Bentin, S., Berg, P., Donchin, E., Hillyard, S. A., Johnson, R., et al. (2000). Guidelines for using human event-related potentials to study cognition: Recording standards and publication criteria. Psychophysiology, 37(2), 127-152.

Pizzagalli, D. A., Peccoralo, L. A., Davidson, R. J., \& Cohen, J. D. (2006). Resting anterior cingulate activity and abnormal responses to errors in subjects with elevated depressive symptoms: a 128-channel EEG study. Human Brain Mapping, 27(3), 185201.

Pourtois, G., Dan, E. S., Grandjean, D., Sander, D., \& Vuilleumier, P. (2005). Enhanced extrastriate visual response to bandpass spatial frequency filtered fearful faces: Time course and topographic evoked-potentials mapping. Hum Brain Mapp, 26(1), 65-79.

Pourtois, G., De Pretto, M., Hauert, C. A., \& Vuilleumier, P. (2006). Time course of brain activity during change blindness and change awareness: performance is predicted by neural events before change onset. J Cogn Neurosci, 18(12), 2108-2129.

Pourtois, G., Delplanque, S., Michel, C., \& Vuilleumier, P. (2008). Beyond conventional event-related brain potential (ERP): exploring the time-course of visual emotion processing using topographic and principal component analyses. Brain Topogr, 20(4), 265-277.

Pourtois, G., Peelen, M. V., Spinelli, L., Seeck, M., \& Vuilleumier, P. (2007). Direct intracranial recording of body-selective responses in human extrastriate visual cortex. Neuropsychologia, 45(11), 2621-2625.

Pourtois, G., Thut, G., Grave de Peralta, R., Michel, C., \& Vuilleumier, P. (2005). Two electrophysiological stages of spatial orienting towards fearful faces: early temporo- 
parietal activation preceding gain control in extrastriate visual cortex. Neuroimage, 26(1), 149-163.

Pourtois, G., Vocat, R., N'Diaye, K., Spinelli, L., Seeck, M., \& Vuilleumier, P. (2010). Errors recruit both cognitive and emotional monitoring systems: simultaneous intracranial recordings in the dorsal anterior cingulate gyrus and amygdala combined with fMRI. Neuropsychologia, 48(4), 1144-1159.

Ridderinkhof, K. R., Nieuwenhuis, S., \& Bashore, T. R. (2003). Errors are foreshadowed in brain potentials associated with action monitoring in cingulate cortex in humans. Neurosci Lett, 348(1), 1-4.

Ridderinkhof, K. R., Nieuwenhuis, S., \& Braver, T. S. (2007). Medial frontal cortex function: an introduction and overview. Cognitive, Affective \& Behavioral Neuroscience, 7(4), 261-265.

Ridderinkhof, K. R., Ullsperger, M., Crone, E. A., \& Nieuwenhuis, S. (2004). The role of the medial frontal cortex in cognitive control. Science, 306(5695), 443-447.

Robbins, T. W. (2007). Shifting and stopping: fronto-striatal substrates, neurochemical modulation and clinical implications. Philos Trans R Soc Lond B Biol Sci, 362(1481), 917-932.

Rowe, J. B., Toni, I., Josephs, O., Frackowiak, R. S., \& Passingham, R. E. (2000). The prefrontal cortex: response selection or maintenance within working memory? Science, 288(5471), 1656-1660.

Rushworth, M. F., Paus, T., \& Sipila, P. K. (2001). Attention systems and the organization of the human parietal cortex. J Neurosci, 21(14), 5262-5271.

Rushworth, M. F., \& Taylor, P. C. (2006). TMS in the parietal cortex: updating representations for attention and action. Neuropsychologia, 44(13), 2700-2716.

Scheffers, M. K., Coles, M. G., Bernstein, P., Gehring, W. J., \& Donchin, E. (1996). Eventrelated brain potentials and error-related processing: an analysis of incorrect responses to go and no-go stimuli. Psychophysiology, 33(1), 42-53.

Sekihara, K., Sahani, M., \& Nagarajan, S. S. (2005). Localization bias and spatial resolution of adaptive and non-adaptive spatial filters for MEG source reconstruction. Neuroimage, 25(4), 1056-1067.

Silva, L., Amitai, Y., \& Connors, B. (1991). Intrinsic oscillations of neocortex generated by layer 5 pyrimidal neurons. Science, 251, 432-435.

Super, H., van der Togt, C., Spekreijse, H., \& Lamme, V. A. (2003). Internal state of monkey primary visual cortex (V1) predicts figure-ground perception. J Neurosci, 23(8), 34073414.

Tibshirani, R., Walther, G., \& Hastie, T. (2001). Estimating the number of clusters in a data set via the gap statistic. Journal of the Royal Statistical Society Series B-Statistical Methodology, 63, 411-423.

Ullsperger, M., \& von Cramon, D. Y. (2001). Subprocesses of performance monitoring: a dissociation of error processing and response competition revealed by event-related fMRI and ERPs. Neuroimage, 14(6), 1387-1401.

van Veen, V., \& Carter, C. S. (2006). Error detection, correction, and prevention in the brain: a brief review of data and theories. Clinical EEG \& Neuroscience, 37(4), 330-335.

van Veen, V., Cohen, J. D., Botvinick, M. M., Stenger, V. A., \& Carter, C. S. (2001). Anterior cingulate cortex, conflict monitoring, and levels of processing. Neuroimage, 14(6), 1302-1308.

Vocat, R., Pourtois, G., \& Vuilleumier, P. (2008). Unavoidable errors: a spatio-temporal analysis of time-course and neural sources of evoked potentials associated with error processing in a speeded task. Neuropsychologia, 46(10), 2545-2555. 
Weissman, D. H., Roberts, K. C., Visscher, K. M., \& Woldorff, M. G. (2006). The neural bases of momentary lapses in attention. Nat Neurosci, 9(7), 971-978.

Woldorff, M. G., Hazlett, C. J., Fichtenholtz, H. M., Weissman, D. H., Dale, A. M., \& Song, A. W. (2004). Functional parcellation of attentional control regions of the brain. $J$ Cogn Neurosci, 16(1), 149-165.

Yeung, N., Cohen, J. D., \& Botvinick, M. M. (2004). The neural basis of error detection: conflict monitoring and the error-related negativity. Psychol Rev, 111(4), 931-959.

\section{Figures legend}

Figure1. Grand average ERP data for Fast Hits (epoched -500/+500 ms around the response RT). (A) Butterfly presentation of all 61 recording channels, plus the GFP waveform superimposed (black waveform). The ERP waveform for the electrode FCz was highlighted in red. "P" indicated the peak of the motor preparatory activity, and only during this motor preparation stage did the GFP reliably increase during the pre-response time period (see GFP results). (B) Dissimilarity values computed during the $-500 /+500 \mathrm{~ms}$ time interval around the response, showing a clear peak (indicated by the arrow and suggesting the occurrence of a main topographic change) during the $500 \mathrm{~ms}$ pre-response time period, while the GFP (amplitude of ERP signal) was visibly close to the zero baseline during this time interval. Another dissimilarity peak was also detected closer to the onset of the response, likely reflecting the transition between motor preparation and motor execution. (C) The sequence of successive topographic maps was displayed as a function of GFP (see also Fig. 1A and Lehmann \& Skrandies, 1980). The dominant segments were expressed as standard 2-D topographic maps, color-coded using the amplitude value at each channel, normalized by the GFP (see Lehmann \& Skrandies, 1980). A spatial cluster analysis confirmed that this first peak of dissimilarity corresponded to a genuine topographic change, as revealed by a substantial modification of the electric field configuration (i.e., an initial occipital baseline map, encircled in the orange frame, suddenly transformed into a transition occipito-parietal topographic map, encircled in the red frame, before this transition map eventually turned to a motor preparation stage where the GFP showed some reliable increase). (D) Dissimilarity values computed during the $-500 /+500 \mathrm{~ms}$ time interval around the response (same as B), but when the number of trials was matched between fast hits and errors, as achieved by computing a new ERP waveform for fast hits including a substantially lower number of trials.

Figure2. Grand average ERP data for Errors (-500/+500 $\mathrm{ms}$ around the response). (A) Butterfly presentation of all 61 recording channels, plus the GFP waveform (black waveform). The ERP waveform for the electrode $\mathrm{FCz}$ was highlighted in red. "P" indicated the peak of the motor preparatory activity. Prominent ERN and Pe ERP components were generated following the onset of the response. (B) Dissimilarity values computed during the $500 /+500 \mathrm{~ms}$ time interval around the response. No obvious and main peak of dissimilarity could be detected during the $500 \mathrm{~ms}$ pre-response time period (compare to Fig. 1B and Fig. $3 \mathrm{~B}$ ), with the exception of a peak occurring close to the onset of the response, and likely corresponding to a topographic transition between motor preparation and motor execution. 
This peak of dissimilarity was also found for Fast hits (see Fig. 1B) and for Slow hits (see Fig. 3B). There were multiple increases of map dissimilarity for Errors during the $500 \mathrm{~ms}$ preresponse time period. (C) A cluster analysis confirmed that the occipito-parietal transition map found for Fast hits (Fig. 1C) and Slow hits (Fig. 3C) was not detected for Errors, although the initial occipital map was the same for Errors, relative to the two other conditions.

Figure3. Grand average ERP data for Slow Hits $(-500 /+500 \mathrm{~ms}$ around the response). (A) Butterfly presentation of all 61 recording channels, plus the GFP waveform (black waveform). The ERP waveform for the electrode $\mathrm{FCz}$ was highlighted in red. "P" indicated the peak of the motor preparatory activity, which was clearly occurring earlier for Slow hits compared to either Fast hits (Fig. 1A) or Errors (Fig. 2A). (B) Dissimilarity values computed during the $-500 /+500 \mathrm{~ms}$ time interval around the response, showing a clear peak (indicated by the arrow and suggesting the occurrence of a main topographic change) during the $500 \mathrm{~ms}$ pre-response time period, while the GFP (amplitude of ERP signal) was close to the zero baseline. Another dissimilarity peak was also detected closer to the onset of the response, for the transition between motor preparation and motor execution. (C) A cluster analysis confirmed that this initial peak of dissimilarity corresponded to a genuine topographic change, as revealed by a substantial modification of the electric field distribution (i.e., an initial occipital baseline map, encircled in the orange frame, suddenly transformed into a transition occipito-parietal topographic map, encircled in the red frame, before this transition map eventually turned to a motor preparation stage; hence showing a similar sequence of topographic events relative to Fast hits).

Figure4. Stimulus-locked grand average ERP data. (A) ERP data (electrode FCz) for the different experimental conditions. The vertical dotted line corresponded to the onset of the response (mean RTs for fast hits and errors). No obvious amplitude or latency difference could be detected across conditions during the $250 \mathrm{~ms}$ time interval following stimulus onset. By contrast, a clear ERN component was visible for response errors in this stimulus-locked ERP analysis, peaking $300 \mathrm{~ms}$ post-stimulus onset (see Falkenstein et al., 2000). For correct rejections, a clear nogo P3 component (Kok, 1986) was also recorded $\sim 370$ ms post-stimulus onset. (B) Results of the spatial cluster analysis run on the $500 \mathrm{~ms}$ time interval following stimulus onset. A solution with 7 dominant maps explained $94 \%$ of the variance (see results). For each condition, the sequence of successive topographic maps (labeled with a number between 1 and 7) was displayed as a function of GFP (see Lehmann \& Skrandies, 1980). This analysis confirmed the lack of significant topographic difference across conditions during the $250 \mathrm{~ms}$ time interval following stimulus onset. The vertical dotted line corresponded to the onset of the response. (C) The 7 dominant segments were expressed as standard 2-D topographic maps, color-coded using the amplitude value at each channel, normalized by the GFP (see Lehmann \& Skrandies, 1980). (D) ERP data (electrode Oz) for the different experimental conditions. A visual N1 was clearly visible for all conditions, with similar amplitude (and latency) for errors and correct (fast) hits. No obvious amplitude or latency 
difference could be detected across conditions during the $250 \mathrm{~ms}$ time interval following stimulus onset in this occipital electrode position.

Figure5. Statistical results of the spatial cluster analysis (i.e., fitting of dominant map configurations identified at the group level back to individual ERP data). (A) During the time interval corresponding to the topographic transition (from 210 to $90 \mathrm{~ms}$ before motor response), I found a highly significant $(\mathrm{p}<.001)$ interaction between map configurations $(2$ levels) and conditions (3 levels) for the global explained variance (GEV; mean \pm 1 S.E.M.). Whereas the two distinctive maps were equally present for Errors (no clear topographic transition), by comparison both for Fast hits and Slow Hits I found that the transition occipitoparietal map had a larger global explained variance (GEV) than the initial occipital map, indicating a genuine change of electric field configuration for these two conditions (see also results). The GEV for the initial occipital topographic map was not different for Fast hits and Errors. (B) A correlation analysis showed, across the 16 participants, a significant negative relationship $[\mathrm{r}(16)=-.52, \mathrm{p}=.041]$ between the duration of the transition topographic map and the (absolute) magnitude of the ERN component following incorrect responses (errors). The shorter the transition map during the pre-response time period, the larger the ERN component following the incorrect response. After removing the data of one outlier (red diamond), this negative correlation became even more significant $[\mathrm{r}(15)=-.70, \mathrm{p}=.004]$. The linear regression line $(y=-10.62 x+95,61)$ computed for the 16 data points was presented.

Figure6. Source localization results, based on sLORETA (R. D. Pascual-Marqui, 2002). (A) The neural generators of the initial (baseline) map, shared across all three conditions (see Figs. 1C, 2C and 3C), were primarily localized within medial regions of the occipital cortex (Brodmann Area 19; Cuneus; right: $+5 \mathrm{x},-90 \mathrm{y},+30 \mathrm{z}$; left: $-4 \mathrm{x},-92 \mathrm{y},+24 \mathrm{z}$ ), consistent with the early sensory processing of the imperative visual stimulus in this task. (B) By contrast, the brain sources of the transition map were mainly found in more dorsal cortical regions, at the border between the cuneus and precuneus, in the parietal cortex (Brodmann Area 7; right: 10x, -80y, +43z; left: $-8 \mathrm{x},-80 \mathrm{y},+40 \mathrm{z}$ ). (C) A direct statistical comparison (paired t-test) performed between Fast Hits and Errors in this inverse solution space during the pre-response time period where the main topographic transition was found to be attenuated for Errors (170$150 \mathrm{~ms}$ before response) revealed a highly significant $(\mathrm{p}<.001)$ activation for Fast hits relative to Errors, circumscribed within the superior parietal lobule/precuneus (right: $5 \mathrm{x},-70 \mathrm{y},+55 \mathrm{z}$, tvalue: 5.92, $\mathrm{p}<.001$; left: $-5 \mathrm{x},-70 \mathrm{y},+55 \mathrm{z}, \mathrm{t}$-value: 5.50, p<.001). (D) Source localization results for the ERN component confirmed that neural generators of this error-related ERP component were primarily localized in the medial frontal gyrus (right: $+5 \mathrm{x},-22 \mathrm{y},+57 \mathrm{z}$; left: $4 \mathrm{x},-22 \mathrm{y},+55 \mathrm{z}$ ), extending more ventrally towards the rostral cingulate gyrus (right: $+9 \mathrm{x}$, $17 y,+45 z$; left: $-9 x,-17 y,+45 z)$, consistent with earlier source localization results for the ERN component (see Dehaene et al., 1994; Herrmann et al., 2004; Pizzagalli et al., 2006; O'Connell et al., 2007; Vocat et al., 2008). 
Supplementary Figure1. (A) A control analysis was carried out to assess whether the occurrence of errors (i.e. false alarms) varied with the delay/SOA (between the black arrow/cue and the changing arrow/target) or not. This SOA varied randomly between 1000 and $2000 \mathrm{~ms}$ (with steps of $100 \mathrm{~ms}$ ) on a trial by trial basis. This analysis clearly confirmed a lack of systematic relationship between the cue-target interval and the prevalence of errors. Errors were distributed evenly across the different (and randomized) SOAs used. (B) An additional control analysis was also performed to look at the RT distribution for errors, relative to fast hits, and eventually ascertain a reasonable overlap between these two RT distributions. The RT distribution for errors was found to tightly overlap with that obtained for fast hits, confirming that errors were comparable to fast hits. 
A 8

FIGURE 1

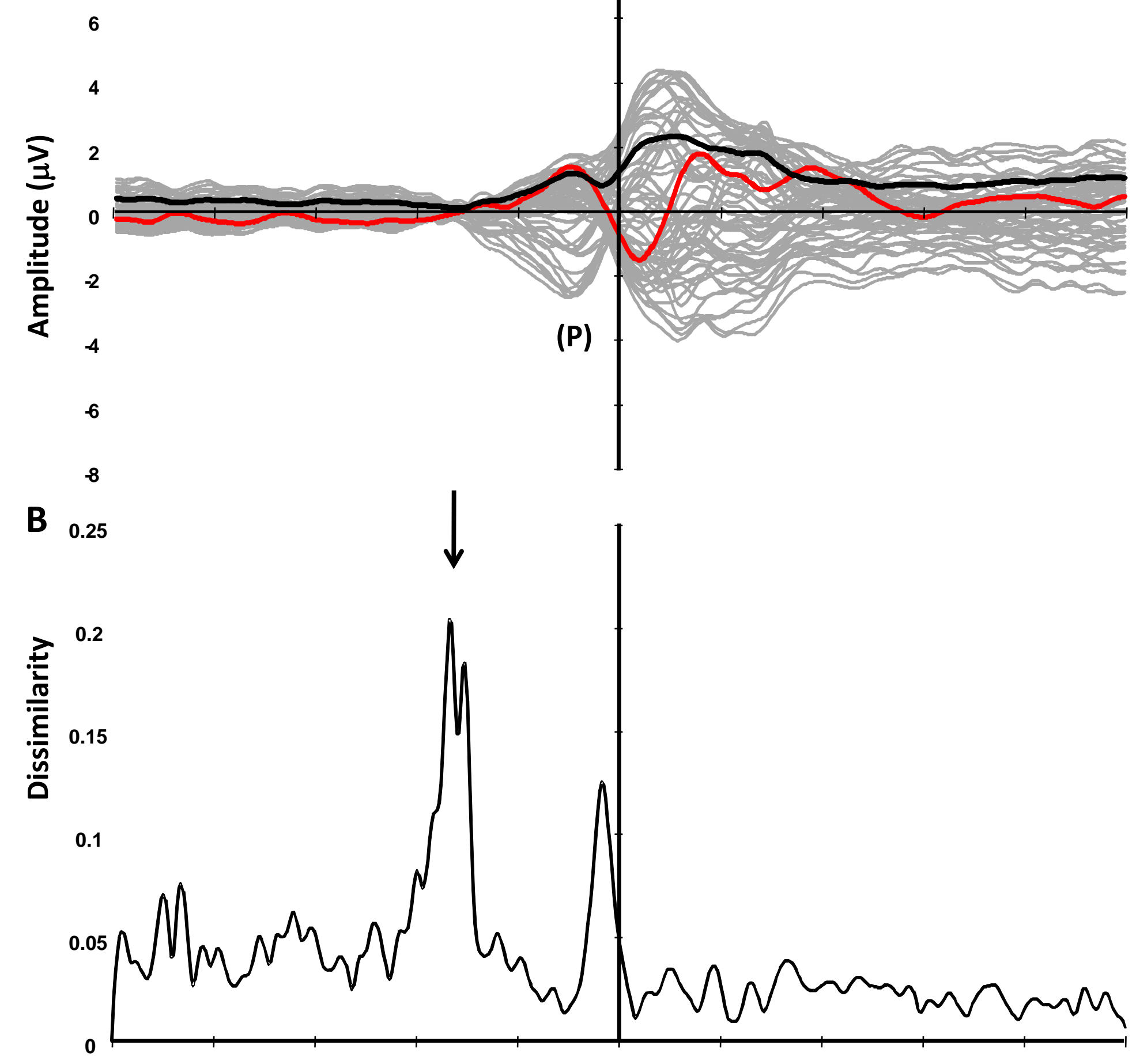

c

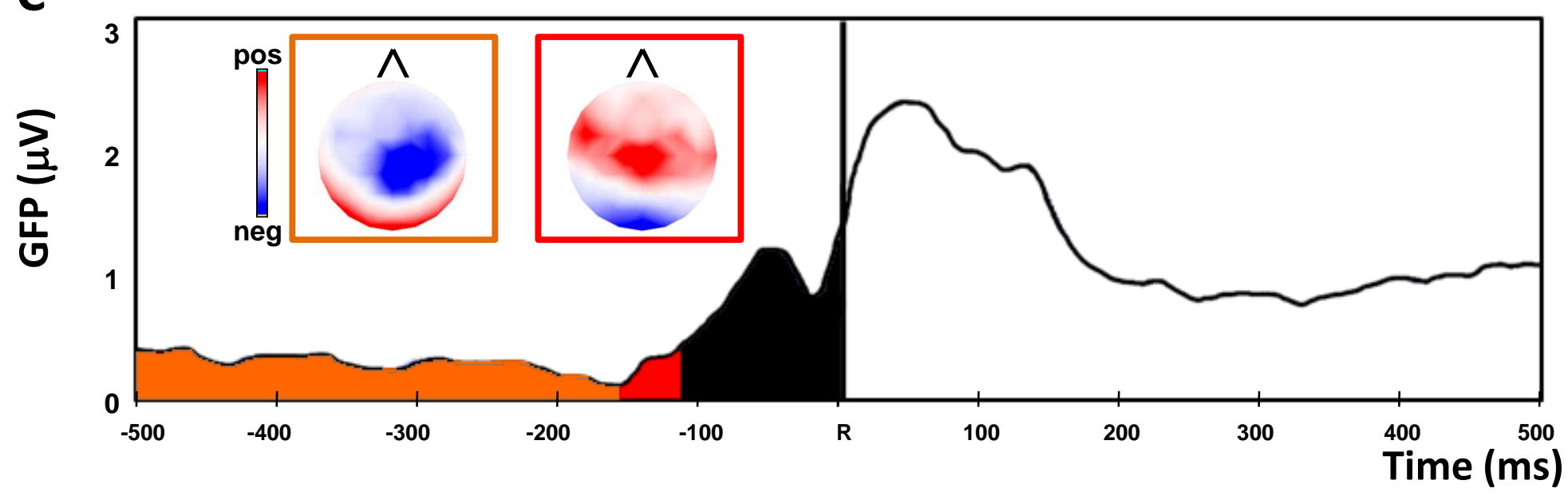


D

FIGURE 1

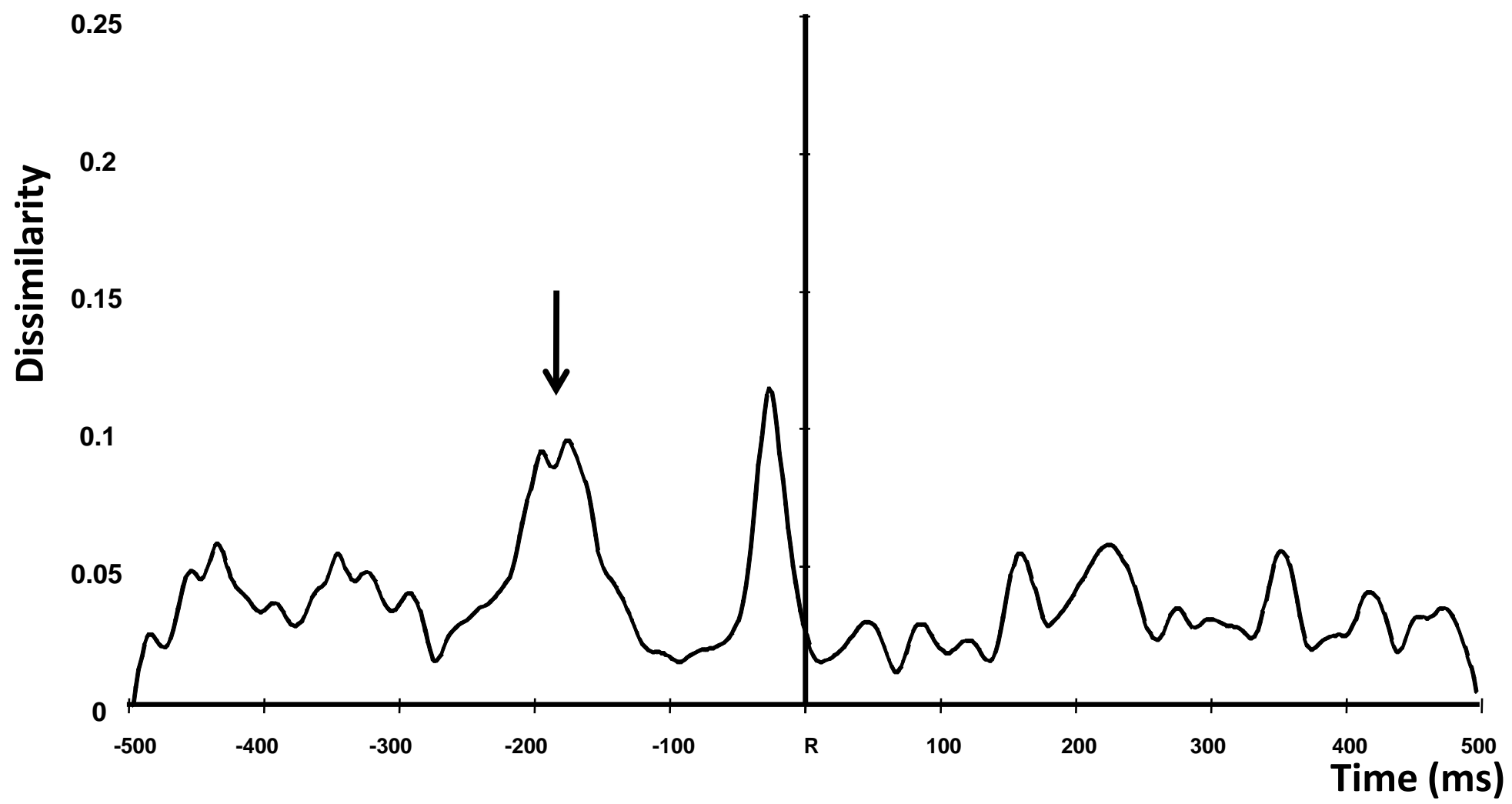


$\begin{array}{llll}A_{8} & \text { (Pe) FIGURE } 2\end{array}$

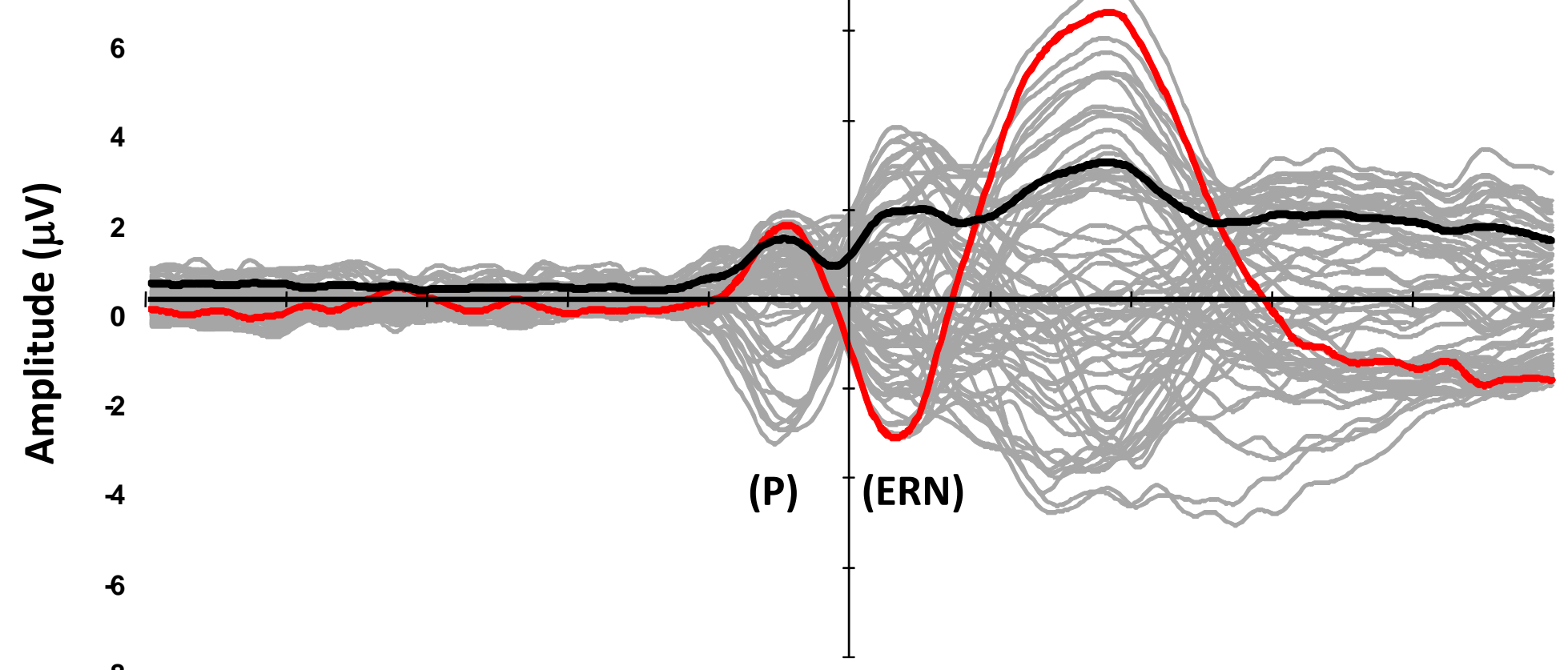

$\begin{array}{lr}\text { B } & 8 \\ & 0.25\end{array}$

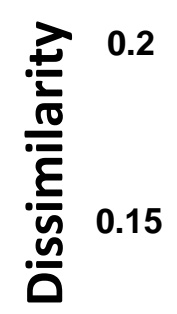

c

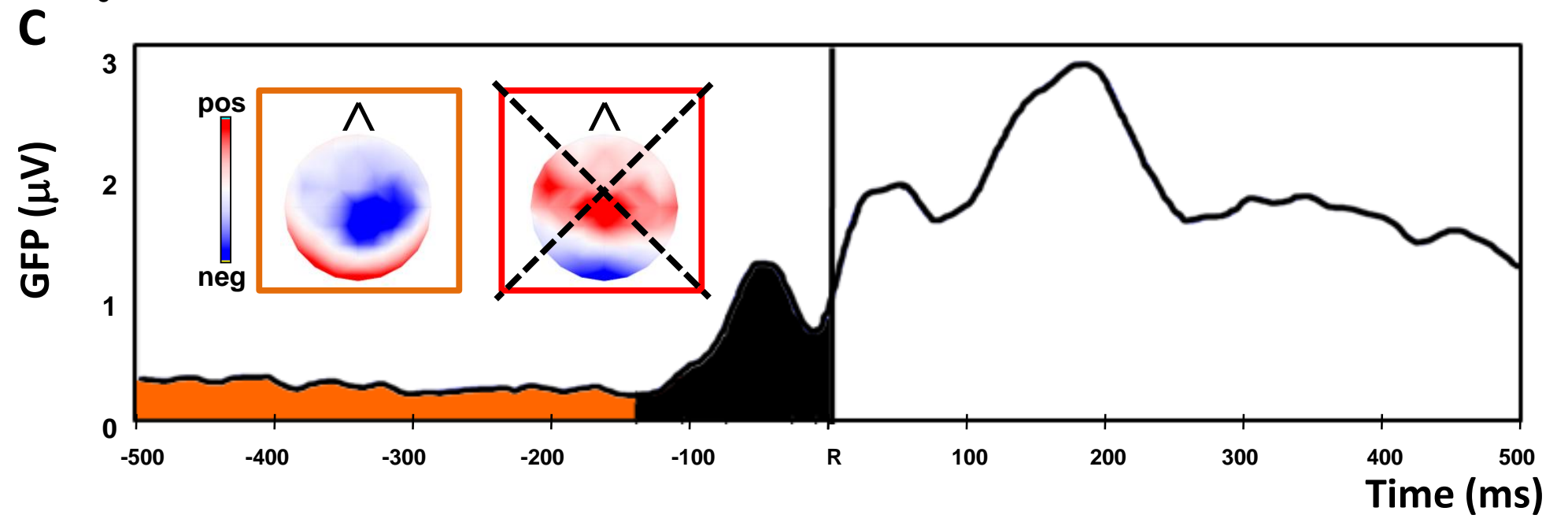


A 8

FIGURE 3
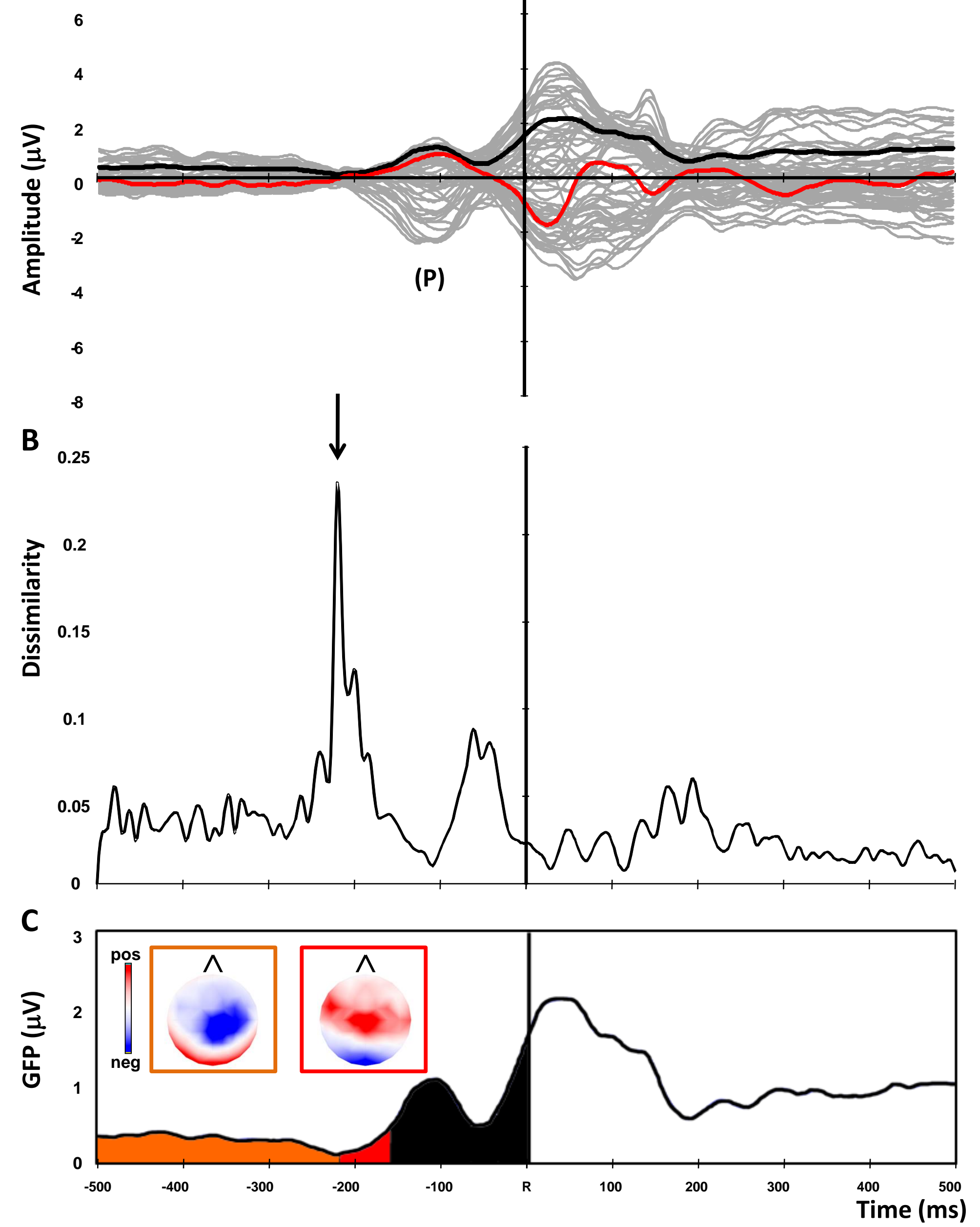


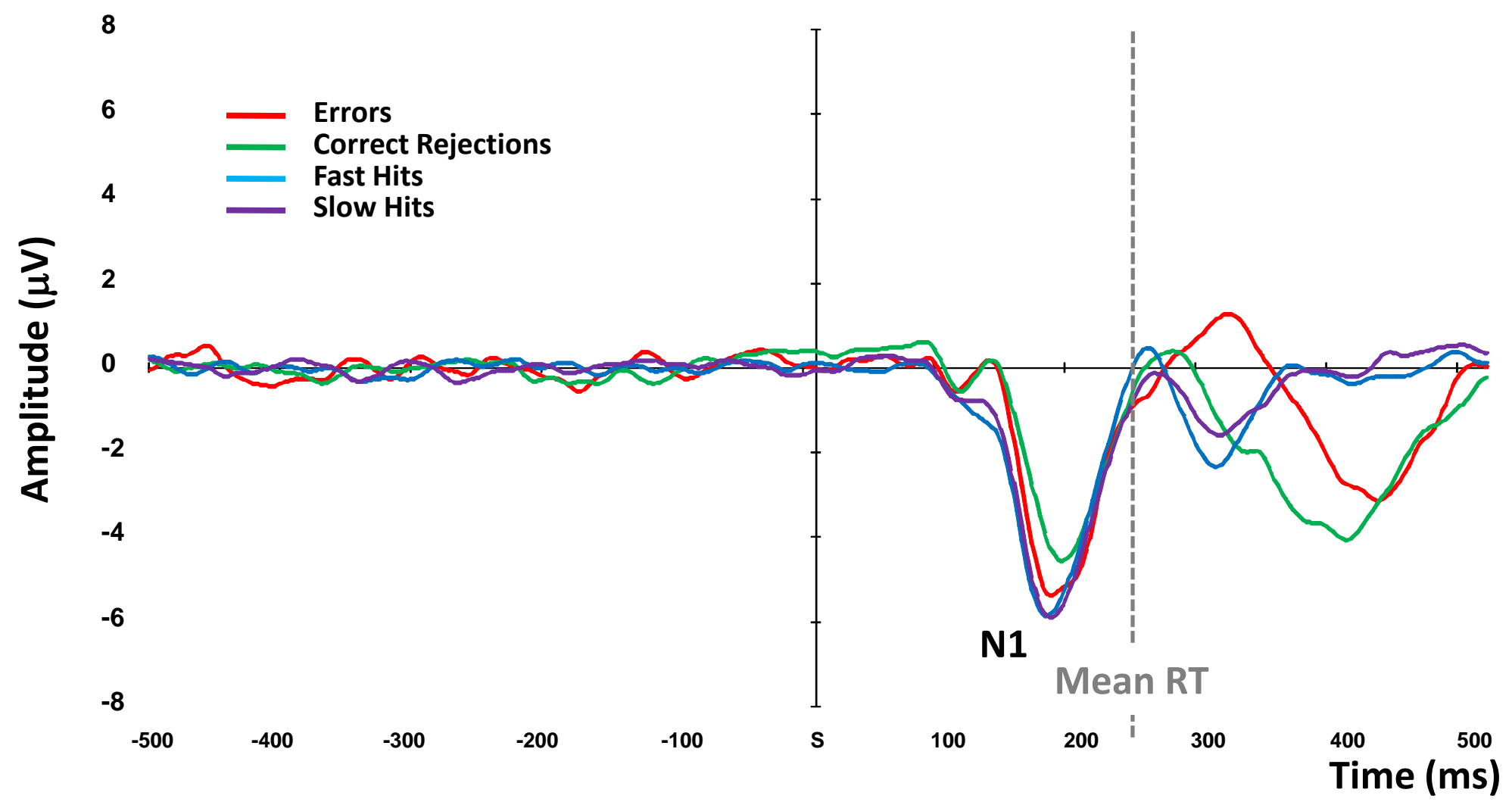


A

FIGURE 5

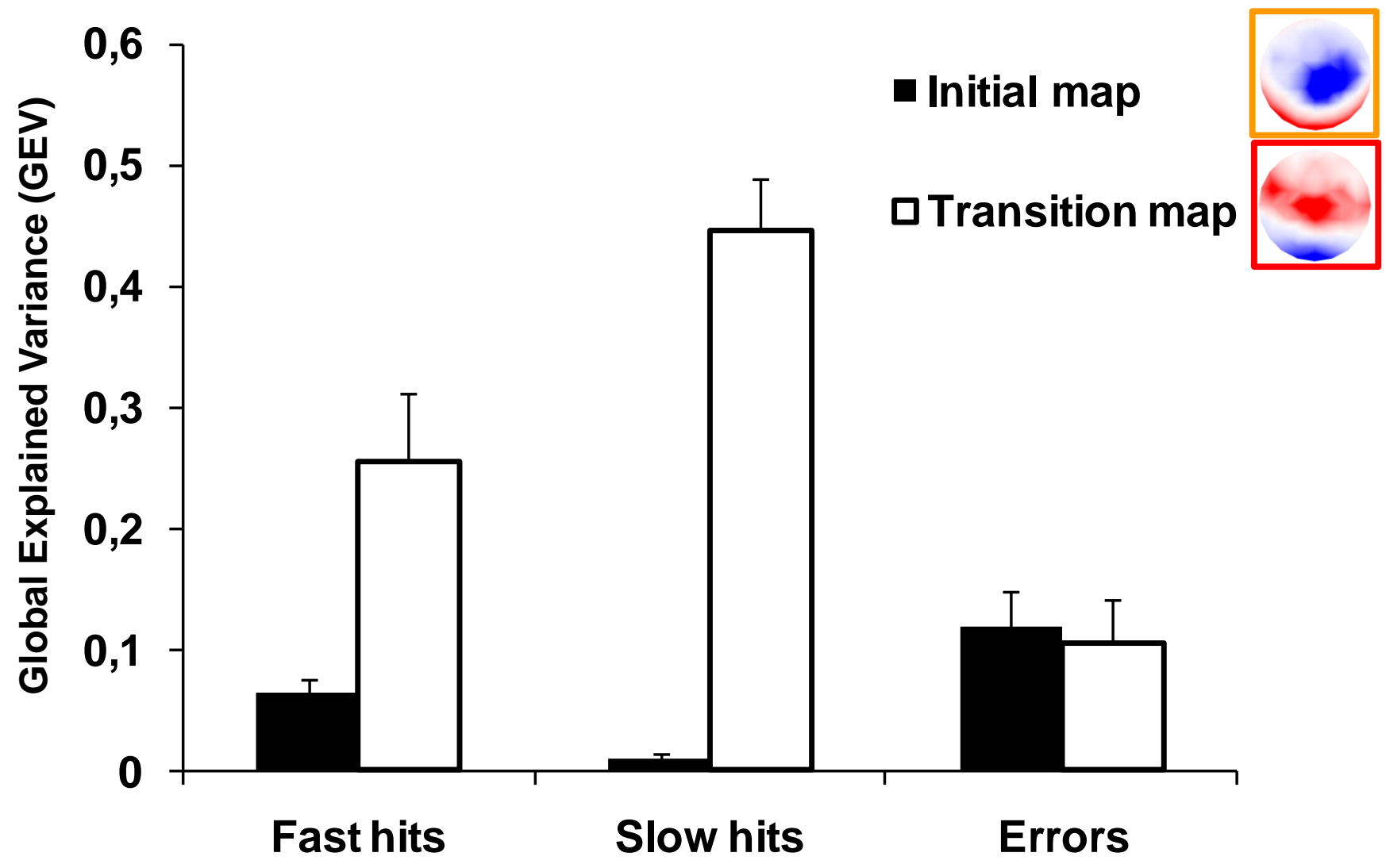

B

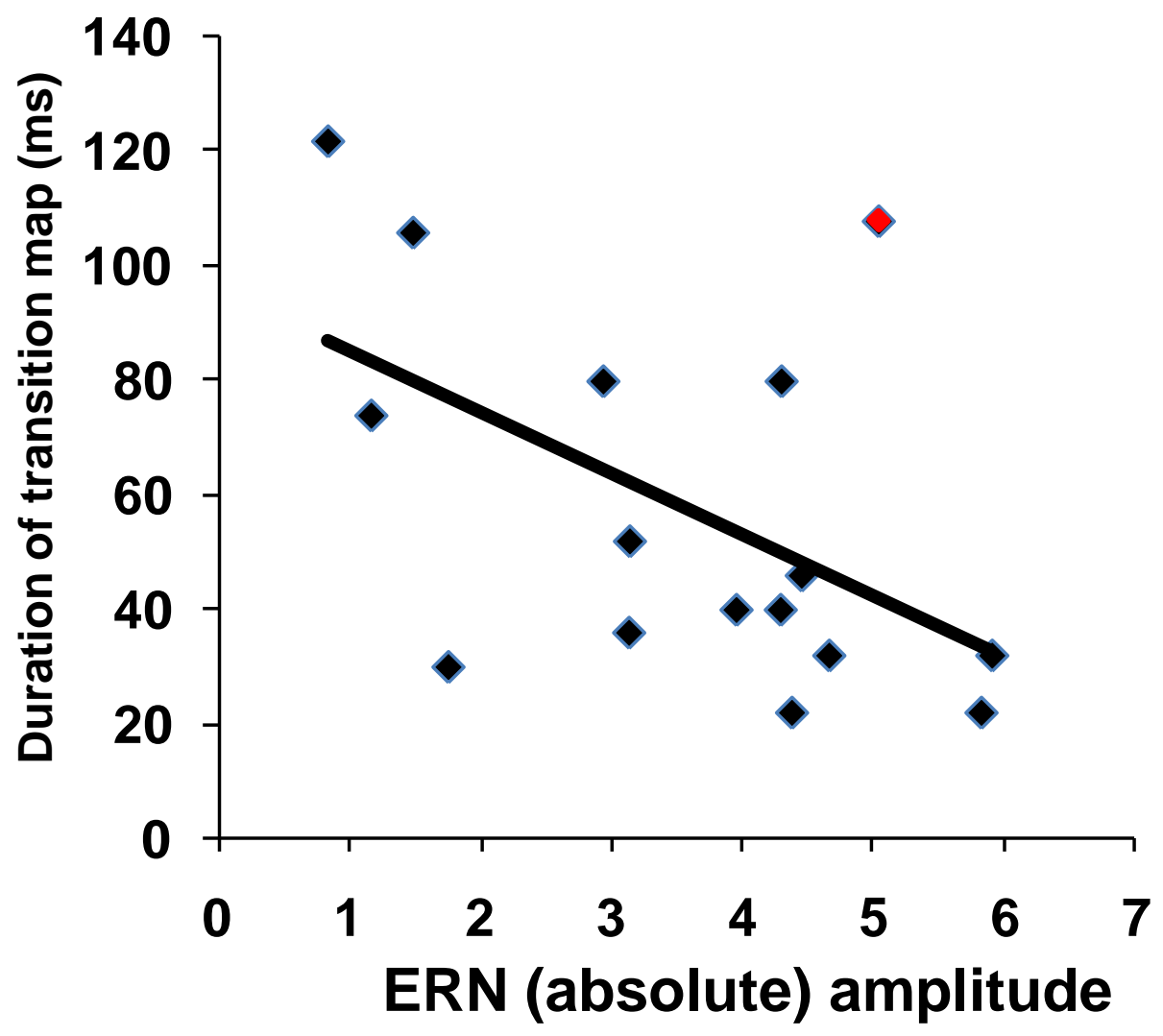


A

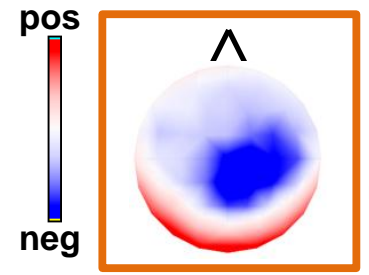

Initial

map

B

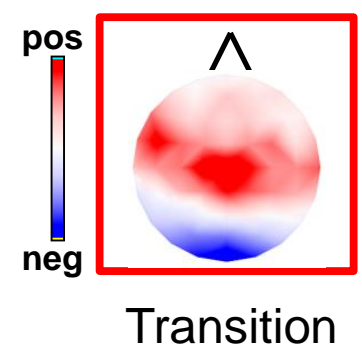

map
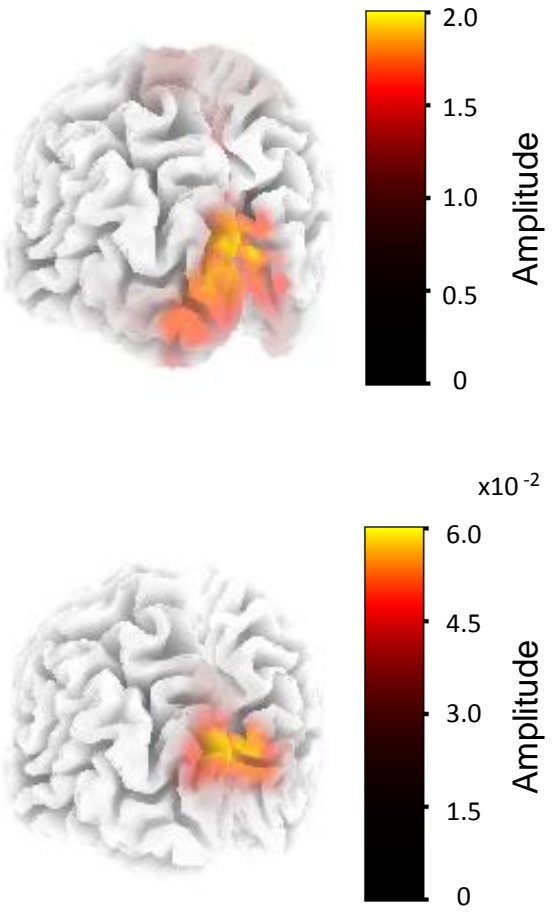

C
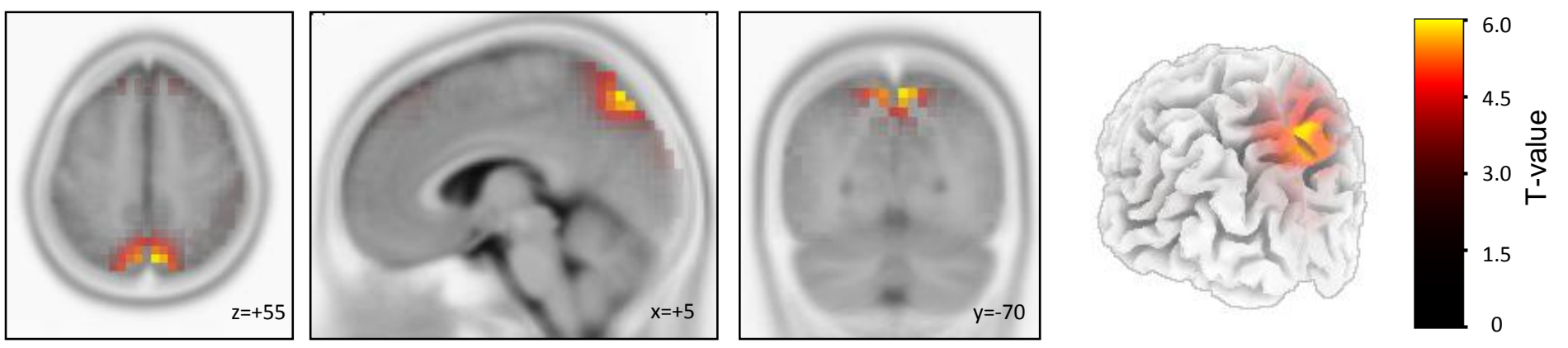

D

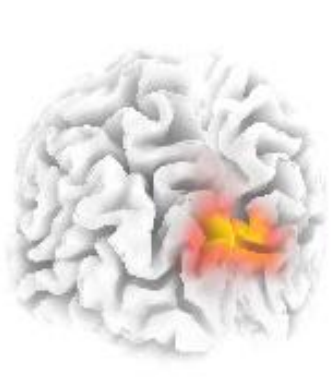


A

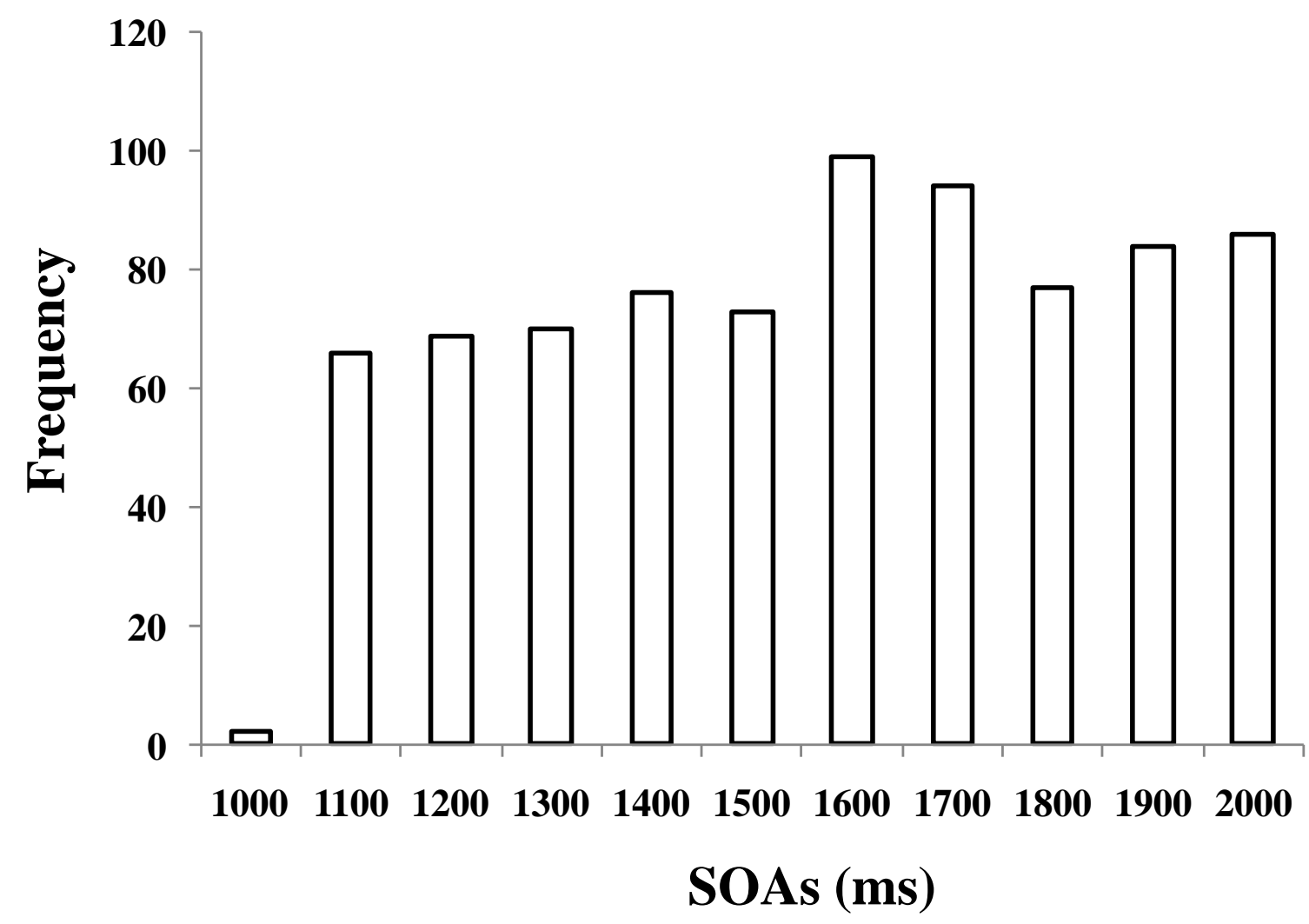

B

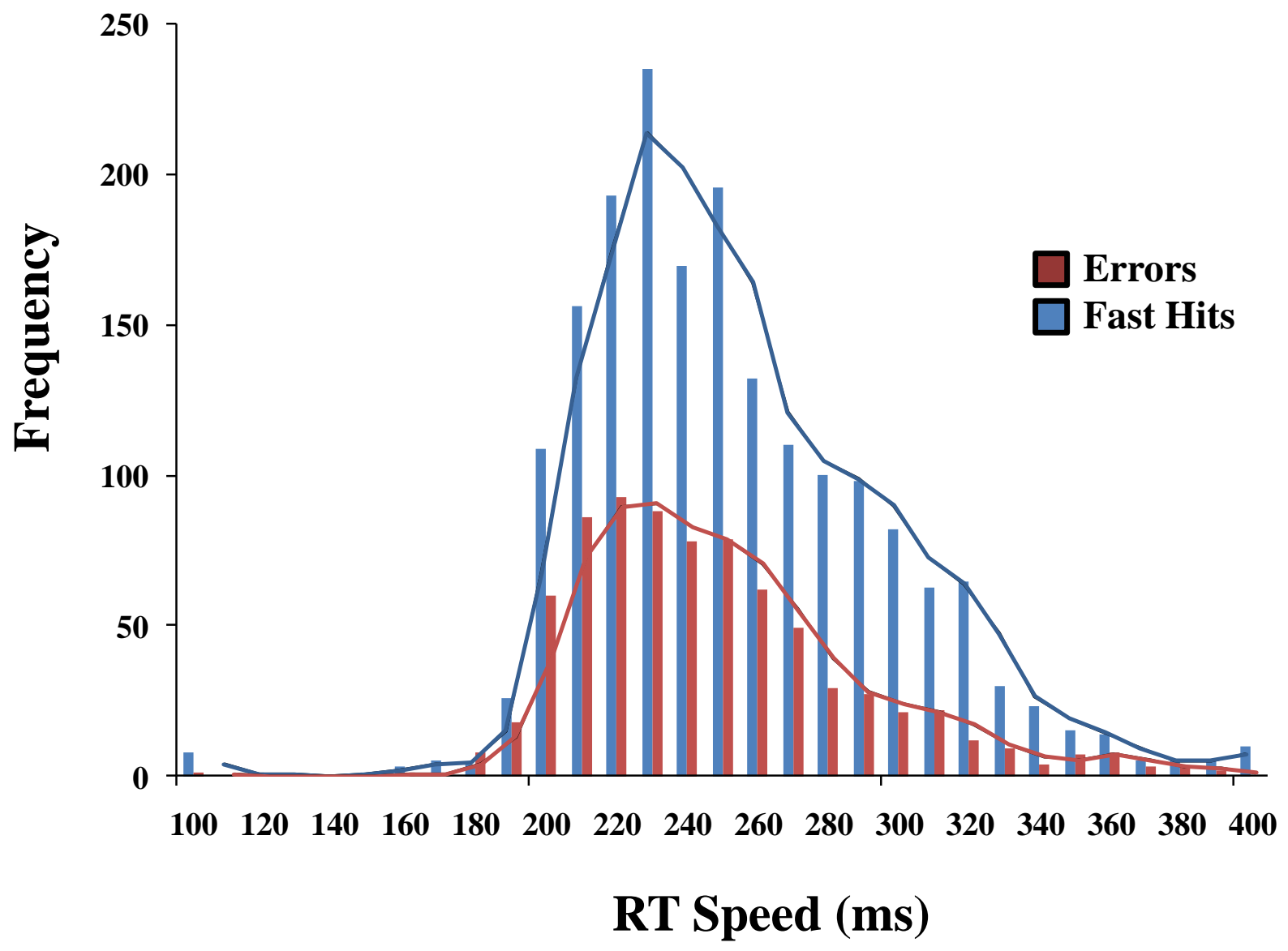

